\title{
DYNAMIC TRADING AND ASSET PRICES: KEYNES VS. HAYEK
}

\author{
Giovanni Cespa ${ }^{1}$ \\ Xavier Vives $^{2}$
}

${ }^{1}$ Cass Business School, CSEF and CEPR

${ }^{2}$ Professor of Economics, Abertis Chair of Regulation, Competition and Public Policy, IESE, and UPF 
The Public-Private Sector Research Center is a Research Center based at IESE Business School. Its mission is to develop research that analyses the relationships between the private and public sectors primarily in the following areas: regulation and competition, innovation, regional economy and industrial politics and health economics.

Research results are disseminated through publications, conferences and colloquia. These activities are aimed to foster cooperation between the private sector and public administrations, as well as the exchange of ideas and initiatives.

The sponsors of the SP-SP Center are the following:

- Accenture

- Ajuntament de Barcelona

- Caixa Manresa

- Departament d' Economia i Coneixement de la Generalitat de Catalunya

- Departament d' Empresa i Ocupació de la Generalitat de Catalunya

- Diputació de Barcelona

- Endesa

- FOBSIC

- Fundació AGBAR

- Institut Català de les Indústries Culturals

- Mediapro

- Sanofi Aventis

- ATM, FGC y TMB

The contents of this publication reflect the conclusions and findings of the individual authors, and not the opinions of the Center's sponsors. 


\title{
Dynamic Trading and Asset Prices: Keynes vs. Hayek *
}

\author{
Giovanni Cespa ${ }^{\dagger}$ and Xavier Vives ${ }^{\ddagger}$ \\ First Version: October 2006 \\ This Version: July 2010
}

\begin{abstract}
We investigate the dynamics of prices, information and expectations in a competitive, noisy, dynamic asset pricing equilibrium model with long-term investors. We argue that the fact that prices can score worse or better than consensus opinion in predicting the fundamentals is a product of endogenous short-term speculation. For a given, positive level of residual payoff uncertainty, if noise trade displays low persistence rational investors act like market makers, accommodate the order flow, and prices are farther away from fundamentals compared to consensus. This defines a "Keynesian" region; the complementary region is "Hayekian" in that rational investors chase the trend and prices are systematically closer to fundamentals than average expectations. The standard case of no residual uncertainty and noise trading following a random walk is on the frontier of the two regions and identifies the set of deep parameters for which rational investors abide by Keynes' dictum of concentrating on an asset "long term prospects and those only." The analysis explains how accommodation and trend chasing strategies differ from momentum and reversal phenomena because of the different information sets that investors and an outside observer have.
\end{abstract}

Keywords: Efficient market hypothesis, long and short-term trading, average expectations, higher order beliefs, over-reliance on public information, opaqueness, momentum, reversal.

JEL Classification Numbers: G10, G12, G14

*We thank Patrick Bolton, Paolo Colla, Martin Dierker, Marcelo Fernandes, Bart Frijns, Diego Garcia, Emmanuel Guerre, Carolina Manzano, Marco Pagano, Alessandro Pavan, Joel Peress, Ailsa Roell, Jaume Ventura, Pietro Veronesi, Paolo Vitale, and seminar participants at the Workshop in Industrial Organization and Finance (IESE), NYU (Economics and Stern), the Federal Reserve Bank of New York, Queen Mary University of London, University of Leicester, the HEC-INSEAD-PSE Workshop (Paris), the European University Institute (Florence), LUISS (Rome), Università Bocconi, Università di Venezia, the 2007 FMA European Conference (Barcelona), the CEPR-CREI Conference "Financial Crises: Past, Theory and Future" (Barcelona), the third CSEF-IGIER Symposium on Economics and Institutions (Anacapri), the 2007 ESSFM (Gerzensee), the 2007 EFA (Ljubljana), the 2008 NSF/NBER/CEME Conference on General Equilibrium and Mathematical Economics (Brown University) and the 2010 AFA meeting for helpful comments. Financial support from the Spanish Ministry of Education and Science (project SEJ2005-08263) is gratefully acknowledged. Vives also acknowledges financial support from the European Research Council under the Advanced Grant project Information and Competition (no. 230254); Project Consolider-Ingenio CSD2006-00016 and project ECO2008-05155 of the Spanish Ministry of Education and Science, as well as the Barcelona GSE Research Network.

${ }^{\dagger}$ Cass Business School, CSEF, and CEPR.

IESE Business School and UPF. 


\section{Introduction}

Do investors excessively focus their attention on market aggregate behavior and public information, disregarding their private judgement? Are asset prices aligned with the consensus opinion (average expectations) on the fundamentals in the market? Undeniably, the issues above have generated much debate among economists. In his General Theory, Keynes pioneered the vision of stock markets as beauty contests where investors try to guess not the fundamental value of an asset but the average opinion of other investors, and end up chasing the crowd. ${ }^{1}$ This view tends to portray a stock market dominated by herding, behavioral biases, fads, booms and crashes (see, for example, Shiller (2000)), and goes against the tradition of considering market prices as aggregators of the dispersed information in the economy advocated by Hayek (1945). According to the latter view prices reflect, perhaps noisily, the collective information that each trader has about the fundamental value of the asset (see, for example, Grossman (1989)), and provide a reliable signal about assets' liquidation values.

Keynes distinguished between enterprise, or the activity of forecasting the prospective yield of assets over their whole life, and speculation, or the activity of forecasting the psychology of the market. In the former the investor focuses on the "long-term prospects and those only" while in the latter he tries to anticipate a change in the convention that guides the stock market valuation of actual investments. Keynes thought that in modern stock markets speculation would be king. Recurrent episodes of bubbles or departures of asset prices from fundamental values have the flavor of Keynes' speculation with traders trying to guess what others will do while prices seem far away from average expectations of fundamentals in the market. In fact, a (somewhat simplistic) version of the Efficient Market Hypothesis (EMH) would say that competition among rational investors will drive prices to be centered around the consensus estimate of underlying value given available information. In other words, prices should equal average expectations of value plus noise. ${ }^{2}$

In this paper, we address the tension between the Keynesian and the Hayekian visions in a dynamic finite horizon market where investors, except for noise traders, have no behavioral bias and hold a common prior on the liquidation value of the risky asset. We therefore allow for the possibility that investors concentrate on "long-term prospects and those only" in a rich noisy dynamic rational expectations environment where there is residual uncertainty on the

\footnotetext{
${ }^{1}$ Keynes' vision of the stock market as a beauty contest - i.e., the situation in which judges are more concerned about the opinion of other judges than of the intrinsic merits of the participants in the contest - is vividly expressed in the twelfth chapter of the General Theory: "...professional investment may be likened to those newspaper competitions in which the competitors have to pick out the six prettiest faces from a hundred photographs, the prize being awarded to the Competitor whose choice most nearly corresponds to the average preferences of the competitor as a whole; so that each competitor has to pick, not those faces which he himself finds prettiest, but those which he thinks likeliest to catch the fancy of the other competitors, all of whom are looking at the problem from the same point of view." (Keynes, Ch. 12, General Theory, 1936).

${ }^{2}$ Professional investors attribute considerable importance to the consensus estimate as a guide to selecting stocks. Bernstein (1996) reports how in 1995 Neil Wrigth, chief investment officer of ANB Investment Management \& Trust, introduced a strategy "explicitly designed to avoid the Winner's Curse." Such a strategy was based on the composition of a portfolio from stocks with a narrow trading range, "an indication that [these stocks] are priced around consensus views, with sellers and buyers more or less evenly matched. The assumption is that such stocks can be bought for little more than their consensus valuation."
} 
liquidation value of the asset (so that the collective information of rational investors is not sufficient to recover the fundamentals) and where noise trading follows a general process.

We find that as long as rational investors find it profitable to engage in short-term speculation, the simplistic EMH does not hold in our model. ${ }^{3}$ Furthermore, the fact that prices can be systematically farther away or closer to fundamentals compared to consensus, or that they can display over- or under-reliance on public information and score worse or better than consensus in predicting the fundamentals are all manifestations of the same phenomenon: endogenous short-term speculation. ${ }^{4}$ In a static market investors speculate on the difference between the price and the liquidation value, prices are aligned with their average expectations about this value, and investors put the optimal statistical weight on public information. Thus, in this context the price is just a noisy measure of investors' consensus opinion. In a dynamic market, investors speculate also on short-run price differences. With heterogeneous information, this may misalign prices and investors' average expectations, potentially leading prices either closer or farther away from the fundamentals compared to consensus. Two key deep parameters, the level of residual payoff uncertainty and the degree of persistence of noise trades, determine whether either over- or under-reliance on public information occur. When there is no residual uncertainty on the asset liquidation value and noise trading follows a random walk then prices are aligned with consensus like in a static market. This is one of the boundary cases where rational investors do not have incentives to speculate on short run price movements. For a given, positive level of residual uncertainty, low persistence generates over-reliance; conversely, high noise trades' persistence tends to generate under-reliance on public information. This partitions the parameter space into a Keynesian region, where prices are farther away from fundamentals than average expectations, and a Hayekian region where the opposite occurs. The boundary of these regions reflects Keynes' situation where investors concentrate on "long-term prospects and those only" and where the (simplistic) EMH holds. In the Keynesian region short run price speculation based on market making motives (reversion of the noise trades process) predominates, while in the Hayekian region short run price speculation based on information (trend chasing) predominates. As a consequence we can characterize accommodation and trend chasing strategies in a model with rational investors and study how do they map to momentum (recent performance tends to persist in the near future) and reversal (a longer history of performance tends to revert).

The intuition for our results is as follows. In a dynamic market, the relationship between price and fundamentals depends both on the quality of investors' information and on their reaction to the aggregate demand. Suppose an investor observes a positive signal and faces a high demand for the asset. Upon the receipt of good news he increases his long position in

\footnotetext{
${ }^{3}$ It should be no surprise that in a noisy rational expectations equilibrium prices may be systematically closer or farther away from the fundamentals compared with investors' average expectations. This result depends on the relative weights that in equilibrium traders put on private and public information and, obviously, could not arise in a fully revealing equilibrium where the price coincides with the liquidation value.

${ }^{4}$ Over-reliance on public information may have deleterious welfare consequences (see, e.g., Vives (1997), Morris and Shin (2002), and Angeletos and Pavan (2007)). In this paper we stay within the bounds of a positive analysis.
} 
the asset. On the other hand, his reaction to high asset demand is either to accommodate it, counting on a future price reversal - thereby acting as a "market-maker" - or to follow the market and further increase his long position anticipating an additional price rise (in this way "chasing" the trend). The more likely it is that the demand realization reverts over time, e.g., due to liquidity traders' transient demand, the more likely that the investor will want to accommodate it. Conversely, the more likely it is that the demand realization proxies for a stable trend, e.g., due to the impact of fundamentals information, the more likely that the investor will want to follow the market. ${ }^{5}$ In the former case, the investor's long-term speculative position is partially offset by his market making position. Thus, the impact of private information on the price is partially sterilized by investors' market making activity. This, in turn, loosens the price from the fundamentals in relation to average expectations, yielding over-reliance on public information. Conversely, in the latter case, the investor's reaction to the observed aggregate demand realization reinforces his long-term speculative position. Thus, the impact of private information on the price is enhanced by the investors' trend chasing activity. This tightens the price to the fundamentals in relation to average expectations, and yields under-reliance on public information. ${ }^{6}$

Low noise trades' persistence strengthens the mean reversion in aggregate demand, and tilts investors towards accommodating the aggregate demand. This effect is extreme when the stock of noise traders' demand is independent across periods. ${ }^{7}$ The impact of residual uncertainty over the liquidation value, on the other hand, enhances the hedging properties of future positions, boosting investors' signal responsiveness and leading them to speculate more aggressively on short-run price differences. Thus, depending on the persistence of noise traders' demand, overor under-reliance on public information occurs, respectively yielding the Keynesian and the Hayekian regions. Conversely, when noise traders' demand is very persistent (i.e., when noise trades increments are i.i.d.) and absent residual uncertainty, investors act as in a static market, and the price assigns the optimal statistical weight to public information. This, together with the boundary between the Keynesian and the Hayekian regions, identifies the set of parameter values for which investors concentrate on the asset long term prospects, shying away from short term speculation.

Interestingly, the Keynesian and Hayekian regions can be characterized in terms of investors' consensus opinion about the systematic behavior of future price changes. Indeed, in the Hayekian region, investors chase the market because the consensus opinion is that prices will systematically continue a given trend in the upcoming trading period. In the Keynesian

\footnotetext{
${ }^{5}$ In this case, indeed, the aggregate demand is likely to proxy for upcoming good news that are not yet completely incorporated in the price. There is a vast empirical literature that documents the transient impact of liquidity trades on asset prices as opposed to the permanent effect due to information-driven trades. See e.g. Wang (1994), and Llorente et al. (2002).

${ }^{6}$ Other authors have emphasized the consequences of investors' reaction to the aggregate demand for an asset. For example, Gennotte and Leland (1990) argue that investors may exacerbate the price impact of trades, yielding potentially destabilizing outcomes, by extracting information from the order flow.

${ }^{7}$ Indeed, assuming that the stock of noise trade is i.i.d. implies that the gross position noise traders hold in a given period $n$ completely reverts in period $n+1$. This lowers the risk of accommodating the aggregate demand in any period, as investors can always count on the possibility of unwinding their inventory of the risky asset to liquidity traders in the coming round of trade.
} 
region, instead, investors accommodate the aggregate demand because the consensus opinion is that prices will systematically revert. We illustrate how expected price behavior under the latter metric does not always coincide with a prediction based on the unconditional correlation of returns. This is due to the usual signal extraction problem investors face in the presence of heterogeneous information. Thus, in our setup, depending on the patterns of information arrival, returns can display both reversal and momentum. However, these phenomena are compatible with both the Hayekian and Keynesian equilibrium.

This paper contributes to the recent literature that analyzes the effect of higher order expectations in asset pricing models where investors have differential information, but agree on a common prior over the liquidation value. In a dynamic market with risk averse short-term investors, differential information, and an independent stock of noisy supply across periods Allen, Morris, and Shin (2006) argue that prices are always farther away from fundamentals than traders' average expectations and display over-reliance on public information. We show how Keynesian dynamics can arise with long-term investors and how the properties of the noise trading process affect them. Indeed, in our market investors' short-term horizons arise endogenously. Bacchetta and van Wincoop (2006b) study the role of higher order beliefs in asset prices in an infinite horizon model showing that higher order expectations add an additional term to the traditional asset pricing equation, the higher order "wedge," which captures the discrepancy between the price of the asset and the average expectations of the fundamentals. According to our results, higher order beliefs do not necessarily enter the pricing equation. In other words, for the higher order wedge to play a role in the asset price we need residual uncertainty to affect the liquidation value or noise trade increments predictability when traders have long horizons; Nimark (2007), in the context of Singleton (1987)'s model, shows that under some conditions both the variance and the impact that expectations have on the price decrease as the order of expectations increases.

Other authors have analyzed the role of higher order expectations in models where traders hold different initial beliefs about the liquidation value. Biais and Bossaerts (1998) show that departures from the common prior assumption rationalize peculiar trading patterns whereby investors with low private valuations may decide to buy an asset from investors with higher private valuations in the hope to resell it later on during the trading day at an even higher price. Cao and Ou-Yang (2005) study conditions for the existence of bubbles and panics in a model where investors' opinions about the liquidation value differ. ${ }^{8}$ Banerjee et al. (2006) show that in a model with heterogeneous priors differences in higher order beliefs may induce price drift. In related research, Ottaviani and Sørensen (2009) analyze a static binary prediction market in which investors hold different priors about a relevant event. In this setup, they show that the presence of wealth constraints leads the price to under-react to public information.

The paper also contributes to the literature analyzing asset pricing anomalies within the rational expectations equilibrium paradigm. Biais, Bossaerts and Spatt (2008), in a multiasset, noisy, dynamic model with overlapping generations show that momentum can arise in

\footnotetext{
${ }^{8}$ Kandel and Pearson (1995) provide empirical evidence supporting the non-common prior assumption.
} 
equilibrium. Vayanos and Woolley (2008) present a theory of momentum and reversal based on delegated portfolio considerations. We add to this literature by showing how momentum and reversal relate to price over- and under-reliance on public information.

Finally, our paper is related to the literature emphasizing the existence of "limits to arbitrage." De Long et. al (1990) show how the risk posed by the existence of an unpredictable component in the aggregate demand for an asset can crowd-out rational investors, thereby limiting their arbitrage capabilities. ${ }^{9}$ In our setup, it is precisely the risk of facing a reversal in noise traders' positions that tilts informed investors towards accommodating the aggregate demand. In turn, this effect is responsible for the over-reliance that asset prices place on public information.

The paper is organized as follows: in the next section we present the static benchmark, showing that in this framework the asset price places the optimal statistical weight on public information and is just a noisy version of investors' average expectations. In section 3 we analyze the dynamic model and argue that prices display over- or under-reliance on public information whenever, in the presence of heterogeneous information, investors speculate on short term returns. Section 4 analyzes the implications of our model for return regularities. The final section provides concluding remarks.

\section{A Static Benchmark}

Consider a one-period stock market where a single risky asset with liquidation value $v+\delta$, and a riskless asset with unitary return are traded by a continuum of risk-averse, informed investors in the interval $[0,1]$ together with noise traders. We assume that $v \sim N\left(\bar{v}, \tau_{v}^{-1}\right), \delta \sim N\left(0, \tau_{\delta}^{-1}\right)$, and $\delta$ orthogonal to $v$. Speculators have CARA preferences (denote with $\gamma$ the risk-tolerance coefficient) and maximize the expected utility of their wealth: $W_{i}=(v-p) x_{i} \cdot{ }^{10}$ Prior to the opening of the market every informed investor $i$ obtains private information on $v$, receiving a signal $s_{i}=v+\epsilon_{i}, \epsilon_{i} \sim N\left(0, \tau_{\epsilon}^{-1}\right)$, and submits a demand schedule (generalized limit order) to the market $X\left(s_{i}, p\right)$ indicating the desired position in the risky asset for each realization of the equilibrium price. ${ }^{11}$ Assume that $v$ and $\epsilon_{i}$ are independent for all $i$, and that error terms are also independent across investors. Noise traders submit a random demand $u$ (independent of all other random variables in the model), where $u \sim N\left(0, \tau_{u}^{-1}\right)$. Finally, we make the convention that, given $v$, the average signal $\int_{0}^{1} s_{i} d i$ equals $v$ almost surely (i.e. errors cancel out in the aggregate: $\left.\int_{0}^{1} \epsilon_{i} d i=0\right) .{ }^{12}$ The random term $\delta$ in the liquidation value thus denotes the residual uncertainty affecting the final pay off about which no investor possesses information, and can be used as a proxy for the level of opaqueness that surrounds the value of fundamentals. ${ }^{13}$

\footnotetext{
${ }^{9}$ Kondor (2004) shows that limits to arbitrage also occur in a 2-period model where informed traders have market power.

${ }^{10}$ We assume, without loss of generality with CARA preferences, that the non-random endowment of rational investors is zero.

${ }^{11}$ The unique equilibrium in linear strategies of this model is symmetric.

${ }^{12}$ See Section 3.1 in the Technical Appendix of Vives (2008) for a justification of the convention.

${ }^{13}$ One can think that the actual liquidation value of the asset results from the sum of two, orthogonal, random components: $v$ and $\delta$. The former relates to the "traditional" business of the firm, so that an analyst or an
} 
We denote by $E_{i}[Y], \operatorname{Var}_{i}[Y]$ the expectation and the variance of the random variable $Y$ formed by an investor $i$, conditioning on the private and public information he has: $E_{i}[Y]=$ $E\left[Y \mid s_{i}, p\right], \operatorname{Var}_{i}[Y]=\operatorname{Var}\left[Y \mid s_{i}, p\right]$. Finally, let $\alpha_{E}=\tau_{\epsilon} / \tau_{i}$, where $\tau_{i} \equiv\left(\operatorname{Var}_{i}[v]\right)^{-1}$, denote the optimal statistical weight to private information, and $\bar{E}[v]=\int_{0}^{1} E_{i}[v] d i$.

We will use the above CARA-normal framework to investigate conditions under which the equilibrium price is systematically farther away from the fundamentals compared to investors' average expectations. Similarly as in Allen et al. (2006) this occurs whenever for all $v$,

$$
|E[p-v \mid v]|>|E[\bar{E}[v]-v \mid v]| .
$$

In the market, two estimators of the fundamentals are available: the equilibrium price, $p$, and the average expectation investors hold about $v$ (the "consensus opinion"), $\bar{E}[v]$. The above condition then holds if, for any liquidation value, averaging out the impact of noise trades, the discrepancy between the price and the fundamentals is always larger than that between investors' consensus opinion and the fundamentals. ${ }^{14}$

Interestingly, condition (1) turns out to be satisfied whenever investors assign extra weight to public information compared to the optimal statistical weight in the estimation of $v$. Equivalently, (1) holds if and only if the price displays a weaker linear relationship with the fundamentals compared to investors' average opinion. These conclusions follow immediately from the fact that at a linear equilibrium, for a given private signal responsiveness $a>0$, the price can be expressed as

$$
p=\alpha_{P}\left(v+\frac{1}{a} u\right)+\left(1-\alpha_{P}\right) E[v \mid p]
$$

where $\alpha_{P}=a(1+\kappa) / \gamma \tau_{i}$, and $\kappa \equiv \tau_{\delta}^{-1} \tau_{i}$.

Indeed, owing to normality we know that

$$
E_{i}[v]=\alpha_{E} s_{i}+\left(1-\alpha_{E}\right) E[v \mid p]
$$

where $\alpha_{E} \equiv \tau_{\epsilon} / \tau_{i}$, denotes the optimal statistical weight to private information. Because of our convention, we have

$$
\bar{E}[v]=\alpha_{E} v+\left(1-\alpha_{E}\right) E[v] .
$$

From (2) and (3) we have

$$
p-v=\left(1-\alpha_{P}\right)(E[v \mid p]-v)+\alpha_{P} \frac{1}{a} u, \text { and } \bar{E}[v]-v=\left(1-\alpha_{E}\right)(E[v \mid p]-v),
$$

implying

$$
E[\bar{E}[v]-v \mid v]=\left(1-\alpha_{E}\right)(E[E[v \mid p] \mid v]-v), \text { and } E[p-v \mid v]=\left(1-\alpha_{P}\right)(E[E[v \mid p] \mid v]-v) .
$$

Therefore, condition (1) holds if and only if the equilibrium price displays over-reliance on public information in relation to the optimal statistical weight:

$$
\alpha_{P}<\alpha_{E}
$$

expert can obtain information about it. The latter component, instead, originates from decisions and actions that insiders make and regarding which the market is totally clueless.

${ }^{14}$ That is, if condition (1) holds, the price is more biased than the average expectation in the estimation of the fundamentals. 
As stated above we can also show that (1) holds if and only if the price as an estimator of the fundamentals scores worse than the consensus opinion. To this end, we compute the covariance between the price and fundamentals using (2):

$$
\begin{aligned}
\operatorname{Cov}[p, v] & =\operatorname{Cov}\left[\alpha_{P}\left(v+\frac{\theta}{a}\right)+\left(1-\alpha_{P}\right) E[v \mid p], v\right] \\
& =\alpha_{P} \operatorname{Cov}[v, v]+\left(1-\alpha_{P}\right) \operatorname{Cov}[v, E[v \mid p]] \\
& =\alpha_{P} \frac{1}{\tau_{v}}+\left(1-\alpha_{P}\right)\left(\frac{1}{\tau_{v}}-\frac{1}{\tau}\right),
\end{aligned}
$$

where $\tau \equiv \operatorname{Var}[v \mid p]^{-1}=\tau_{v}+a^{2} \tau_{u}$. Similarly, we can compute the covariance between the consensus opinion and the fundamentals:

$$
\operatorname{Cov}[\bar{E}[v], v]=\alpha_{E} \frac{1}{\tau_{v}}+\left(1-\alpha_{E}\right)\left(\frac{1}{\tau_{v}}-\frac{1}{\tau}\right)
$$

Subtracting (6) from (5) yields

$$
\operatorname{Cov}[p-\bar{E}[v], v]=\frac{\alpha_{P}-\alpha_{E}}{\tau}
$$

proving our claim.

We collect the above results in the following lemma:

Lemma 1. In the static market, the following three conditions are equivalent:

$$
\begin{aligned}
|E[p-v \mid v]| & >|E[\bar{E}[v]-v \mid v]| \\
\alpha_{P} & <\alpha_{E} \\
\operatorname{Cov}[p, v] & <\operatorname{Cov}[\bar{E}[v], v] .
\end{aligned}
$$

In the static model it is easy to verify that a unique equilibrium in linear strategies exists in the class of equilibria with a price functional of the form $P(v, u)$ (see, e.g. Admati (1985), Vives (2008)). The equilibrium strategy of an investor $i$ is given by

$$
X\left(s_{i}, p\right)=\frac{a}{\alpha_{E}}\left(E_{i}[v]-p\right),
$$

where

$$
a=\frac{\gamma \tau_{\epsilon}}{1+\kappa}
$$

denotes the market responsiveness to private information and is given by the unique solution to the cubic equation $\phi(a) \equiv a(1+\kappa)-\gamma \tau_{\epsilon}=0 .{ }^{15}$ From the definition of $\alpha_{P}$ and $\alpha_{E}$, we can verify that

$$
\alpha_{P}<\alpha_{E} \Leftrightarrow a<\frac{\gamma \tau_{\epsilon}}{1+\kappa},
$$

which, given (11), is clearly never satisfied. Therefore, we can conclude that in a static market, condition (4) never holds, and the equilibrium price always assigns the optimal statistical weight to public information. ${ }^{16}$

\footnotetext{
${ }^{15}$ It is easy to verify that $\phi(a)=a^{3} \tau_{u}+a\left(\tau_{v}+\tau_{\epsilon}+\tau_{\delta}\right)-\gamma \tau_{\delta} \tau_{\epsilon}=0$ possesses a unique real solution. Indeed, $\phi(0)=-\gamma \tau_{\delta} \tau_{\epsilon}<0, \phi\left(\gamma \tau_{\epsilon}\right)=a\left(a^{2} \tau_{u}+\tau_{v}+\tau_{\epsilon}\right)>0$, implying that a real solution $a^{*}$ exists in the interval $\left(0, \gamma \tau_{\epsilon}\right)$. Finally, since $\left.\phi^{\prime}(a)\right|_{a=a^{*}}>0$, the result follows.

${ }^{16}$ If $E[u]$ is non null, e.g. if $E[u]=\bar{u}>0$, we have to replace the price $p$ by the price net of the expected noise component $\hat{p}=p-\bar{u} \operatorname{Var}_{i}[v+\delta] / \gamma$. Using this definition it is immediate to verify that also when $\bar{u}>0$, in a static market the equilibrium price assigns the optimal statistical weight to public information.
} 
Remark 1. The model introduced above captures the idea that, collectively taken, rational investors do not know the ex-post liquidation value and is therefore qualitatively equivalent to a market in which investors receive a signal with a common error term (like the one studied by Grundy and McNichols (1989)). To see this, maintaining the informational assumptions of our model, suppose that the ex-post liquidation value is given by $v$ whereas investor $i$ receives a signal $s_{i}=v+\delta+\epsilon_{i}$. Then, it is easy to see that in this model there exists a unique equilibrium in linear strategies in which $X\left(s_{i}, p\right)=\left(a / \alpha_{E}\right)\left(E_{i}[v]-p\right)$, where the optimal statistical weight to private information is given by $\alpha_{E} \equiv\left(\left(\tau_{\epsilon}+a^{2} \tau_{u}\right) \tau_{v}+\tau_{\delta}\left(\tau_{\epsilon}+a^{2} \tau_{u}+\tau_{v}\right)\right)^{-1} \tau_{\delta} \tau_{\epsilon}$, and $a$ is the unique real solution to the cubic $\varphi(a) \equiv a^{3} \tau_{u}+a\left(\tau_{\delta}+\tau_{\epsilon}\right)-\gamma \tau_{\delta} \tau_{\epsilon}=0$. As in our model, $a$ is bounded above by $\gamma \tau_{\epsilon}: a \in\left(0, \gamma \tau_{\epsilon}\right)$. With an improper prior about the liquidation value, $\tau_{v}=0$ and the two models yield exactly the same result. When $\tau_{v}>0$, it is possible to show that in the model with a common error in the signal, investors' responsiveness to private information is always higher than in the model considered here. ${ }^{17}$

Remark 2. There is an alternative, more direct way to verify whether condition (1) is satisfied. Indeed, as investors' aggregate demand is proportional to $\int_{0}^{1}\left(E_{i}[v]-p\right) d i$, imposing market clearing in the above model yields

$$
\int_{0}^{1} x_{i} d i+u=\int_{0}^{1} \frac{a}{\alpha_{E}}\left(E_{i}[v]-p\right) d i+u=0,
$$

and solving for the equilibrium price we obtain

$$
p=\bar{E}[v]+\frac{\alpha_{E}}{a} u
$$

In other words, in equilibrium the price is given by the sum of investors' average expectations and noise (times a constant). As $u$ and $v$ are by assumption orthogonal, we can therefore conclude that in a static setup the price assigns the optimal statistical weight to public information. To obtain over-reliance on public information, we thus need to find conditions under which investors' aggregate demand is no longer proportional to $\bar{E}[v]-p$ and this, in a static context with CARA preferences can never happen.

In the following sections we will argue that price over-reliance on public information can be traced to investors' speculative activity on short-run price movements that makes strategies depart from the solution of the static setup.

\section{A 3-Period Model}

Consider now a 3-period extension of the market considered in the previous section. We assume that any speculator $i \in[0,1]$ has CARA preferences and maximizes the expected utility of his

\footnotetext{
${ }^{17}$ To see this it suffices to note that the responsiveness to private information in our model is given by the unique solution to $\phi(a)=a^{3} \tau_{u}+a\left(\tau_{v}+\tau_{\epsilon}+\tau_{\delta}\right)-\gamma \tau_{\delta} \tau_{\epsilon}=0$, whereas in the presence of a common errror in the signal it is given by the solution to $\varphi(a) \equiv a^{3} \tau_{u}+a\left(\tau_{\epsilon}+\tau_{\delta}\right)-\gamma \tau_{\delta} \tau_{\epsilon}=0$. Now $\phi(0)=\varphi(0)=-\gamma \tau_{\delta} \tau_{\epsilon}<0$, and $\phi^{\prime}(0)=\tau_{v}+\tau_{\epsilon}+\tau_{\delta}>\varphi^{\prime}(0)=\tau_{\epsilon}+\tau_{\delta}$, which together with $\phi^{\prime \prime}(a)=\varphi^{\prime \prime}(a)=6 a \tau_{u}$, implies that the unique solution to $\phi(a)=0$ always lays to the left of the unique solution to $\varphi(a)=0$.
} 
final wealth $W_{i 3}=\left(v-p_{3}\right) x_{i 3}+\sum_{n=1}^{2}\left(p_{n+1}-p_{n}\right) x_{i n}{ }^{18}$ In period $n$ an informed investor $i$ receives a signal $s_{i n}=v+\epsilon_{i n}$, where $\epsilon_{i n} \sim N\left(0, \tau_{\epsilon_{n}}^{-1}\right), v$ and $\epsilon_{i n}$ are independent for all $i, n$ and error terms are also independent both across time periods and investors. Denote with $s_{i}^{n} \equiv\left\{s_{i t}\right\}_{t=1}^{n}$ and $p^{n} \equiv\left\{p_{t}\right\}_{t=1}^{n}$, respectively, the sequence of private signals and prices an investor observes at time $n$. Informed investors submit a demand schedule (generalized limit order) to the market $X_{n}\left(s_{i}^{n}, p^{n-1}, p_{n}\right)$ indicating the desired position in the risky asset for each realization of the equilibrium price.

The stock of noise trades is assumed to follow an $\operatorname{AR}(1)$ process: $\theta_{n}=\beta \theta_{n-1}+u_{n}$, where $u_{n} \sim N\left(0, \tau_{u}^{-1}\right)$ is orthogonal to $\theta_{n-1}$, and $\beta \in[0,1] .{ }^{19}$ To interpret, suppose $\beta<1$, then at any period $n>1$ market clearing involves the $n-1$-th and $n$-th period aggregate demands of informed investors (respectively, $x_{n-1} \equiv \int_{0}^{1} x_{i n-1} d i$, and $x_{n} \equiv \int_{0}^{1} x_{i n} d i$ ), a fraction $1-\beta$ of the demand coming from the $n-1$-th generation of noise traders' who revert their positions, and the demand of the new generation of noise traders. Considering the equilibrium conditions for the first two trading dates, and letting $\Delta x_{2} \equiv x_{2}-x_{1}, \Delta \theta_{2} \equiv \theta_{2}-\theta_{1}=u_{2}+(\beta-1) \theta_{1}$, this implies

$$
\begin{gathered}
x_{1}+\theta_{1}=0 \\
\Delta x_{2}+\Delta \theta_{2}=0 \Leftrightarrow x_{2}+\beta \theta_{1}+u_{2}=0 .
\end{gathered}
$$

Thus, assuming that noise trading follows an $\mathrm{AR}(1)$ process allows to take into account the possibility that only part of the trades initiated by noise traders at time $n$ actually reverts at time $n+1$. The lower (higher) is $\beta$, the higher (lower) is the fraction of period $n$ noise traders who will (will not) revert their positions at time $n+1$, and thus won't (will) be in the market at time $n+1$. Equivalently, for $0 \leq \beta<1$, a high, positive demand from noise traders at time $n$ is unlikely to show up with the same intensity at time $n+1$, implying that $\operatorname{Cov}\left[\Delta \theta_{n}, \Delta \theta_{n+1}\right]<0 .{ }^{20}$ Intuitively, a low $\beta$ is likely to occur when the time between two consecutive trades is large. Conversely, a high $\beta$ depicts a situation in which the time between two consecutive transactions is small, so that investors make repeated use of the market to satisfy their trading needs. ${ }^{21}$

\footnotetext{
${ }^{18} \mathrm{We}$ assume, as before without loss of generality, that the non-random endowment of investors is zero.

${ }^{19}$ Our specification for the demand coming from noise traders is consistent with the following model. Replace noise traders with a measure 1 sector of risk-averse, competitive hedgers who receive a random shock to their endowment. A hedger $i$ at time $n$ receives a shock $\theta_{i n}=\theta_{n}+\eta_{i n}$ where $\eta_{i n}$ is a normally distributed white-noise error, uncorrelated with all the other random variables in the model. If we denote by $\gamma^{U}$ the risk-tolerance of hedgers, then letting $\gamma^{U} \rightarrow 0$ implies that each hedger gets rid of $\theta_{i n}$ in the market place. Owing to the convention that $\int_{0}^{1} \eta_{i n} d i=0$, a.s., this in turn implies that the position hedgers hold at time $n$ is given by $\int_{0}^{1} \theta_{i n} d i=\theta_{n}$, yielding the random component of the aggregate demand that we assume in our model. This is in line with Medrano and Vives (2004), who argue that upon receiving a shock to their endowment, infinitely risk-averse hedgers unwind their exposure to the market, yielding the random component of the aggregate demand for the stock that characterizes the model with noise traders. It is worth noting that even in a static model the presence of hedgers generates multiplicity of linear partially revealing equilibria (see, e.g., Ganguli and Yang (2009) and Manzano and Vives (2010)).

${ }^{20}$ Alternatively, the AR(1) assumption for noise traders' demand can be interpreted as a way to parsimoniously model the existence of a positive feedback in these traders' strategies. To see this, consider a 2-period version of our model, then for $\beta>0$, $\operatorname{Corr}\left[\theta_{2}, \theta_{1}\right]=\beta /\left(1+\beta^{2}\right)^{1 / 2}>0$. For two normal random variables, positively correlation is equivalent to the monotone likelihood ratio property. Therefore, we can conclude that if $\beta>0$ the probability of observing a higher $\theta_{2}$ increases in $\theta_{1}$.

${ }^{21}$ The literature that has dealt with dynamic trading models featuring an $\mathrm{AR}(1)$ process for liquidity posits
} 
Extending the notation adopted in the previous section, we denote by $E_{\text {in }}[Y]=E\left[Y \mid s_{i}^{n}, p^{n}\right]$, $E_{n}[Y]=E\left[Y \mid p^{n}\right]\left(\operatorname{Var}_{i n}[Y]=\operatorname{Var}\left[Y \mid s_{i}^{n}, p^{n}\right], \operatorname{Var}_{n}[Y]=\operatorname{Var}\left[Y \mid p^{n}\right]\right)$, respectively the expectation (variance) of the random variable $Y$ formed by an investor conditioning on the private and public information he has at time $n$, and that obtained conditioning on public information only. Finally, we let $\alpha_{E_{n}}=\sum_{t=1}^{n} \tau_{\epsilon_{t}} / \tau_{i n}$, where $\tau_{i n} \equiv\left(\operatorname{Var}_{i n}[v]\right)^{-1}$ and make the convention that, given $v$, at any time $n$ the average signal $\int_{0}^{1} s_{i n} d i$ equals $v$ almost surely (i.e. errors cancel out in the aggregate: $\left.\int_{0}^{1} \epsilon_{i n} d i=0\right)$.

\subsection{The Equilibrium}

In period $1 \leq n \leq 3$ each informed investor has the vector of private signals $s_{i}^{n}$ available. It follows from Gaussian theory that the statistic $\tilde{s}_{i n}=\left(\sum_{t=1}^{n} \tau_{\epsilon_{t}}\right)^{-1}\left(\sum_{t=1}^{n} \tau_{\epsilon} s_{i t}\right)$ is sufficient for the sequence $s_{i}^{n}$ in the estimation of $v$. An informed investor $i$ in period $n$ submits a limit order $X_{n}\left(\tilde{s}_{i n}, p^{n-1}, \cdot\right)$, indicating the position desired at every price $p_{n}$, contingent on his available information. We will restrict attention to linear equilibria where in period $n$ an investor trades according to $X_{n}\left(\tilde{s}_{i n}, p^{n}\right)=a_{n} \tilde{s}_{i n}-\varphi_{n}\left(p^{n}\right)$, where $\varphi_{n}(\cdot)$ is a linear function of the price sequence $p^{n}$. Let us denote with $z_{n}$ the intercept of the $n$-th period net aggregate demand $\int_{0}^{1} \Delta x_{i n} d i+u_{n}$, where $\Delta x_{i n}=x_{i n}-x_{i n-1}$. The random variable $z_{n} \equiv \Delta a_{n} v+u_{n}$ represents the informational addition brought about by the $n$-th period trading round, and can thus be interpreted as the informational content of the $n$-th period order-flow (where, with a slight abuse of notation, we set $\left.\Delta a_{n} \equiv a_{n}-\beta a_{n-1}\right)$. The following proposition characterizes equilibrium prices and strategies:

Proposition 1. Let $\sum_{t=1}^{n} \tau_{\epsilon_{t}}>0$, at any linear equilibrium of the 3-period market the equilibrium price is given by

$$
p_{n}=\alpha_{P_{n}}\left(v+\frac{\theta_{n}}{a_{n}}\right)+\left(1-\alpha_{P_{n}}\right) E_{n}[v], \quad n=1,2,3
$$

where $\theta_{n}=u_{n}+\beta \theta_{n-1}$. For $n=1,2$, an investor's strategy is given by:

$$
X_{n}\left(\tilde{s}_{i n}, z^{n}\right)=\frac{a_{n}}{\alpha_{E_{n}}}\left(E_{i n}[v]-p_{n}\right)+\frac{\alpha_{P_{n}}-\alpha_{E_{n}}}{\alpha_{E_{n}}} \frac{a_{n}}{\alpha_{P_{n}}}\left(p_{n}-E_{n}[v]\right),
$$

while at time 3 :

$$
X_{3}\left(\tilde{s}_{i 3}, z^{3}\right)=\frac{a_{3}}{\alpha_{E_{3}}}\left(E_{i 3}[v]-p_{3}\right),
$$

where $\alpha_{E_{n}}=\sum_{t=1}^{n} \tau_{\epsilon_{t}} / \tau_{\text {in }}$, and expressions for $\alpha_{P_{n}}$ and $a_{n}$ are provided in the appendix (see equations (42), (60), (78), and (41), (56), (81), respectively). The parameters $\alpha_{P_{n}}$ and $a_{n}$ are positive for $n=2,3$. Numerical simulations show that $\alpha_{P_{1}}>0$ and $a_{1}>0$.

relatively high values for $\beta$. For example, in their analysis of a dynamic FX market, Bacchetta and van Wincoop (2006) model the aggregate exposure to the exchange rate as an AR(1) process and in their numerical simulations assume $\beta=0.8$ (Table 1, p. 564). This assumption is somehow validated by empirical analysis. In a recent paper, Easley et al. (2008) analyze the order arrival process using the daily number of buys and sell orders for 16 stocks over a 15-year time period. Their findings point to a highly persistent process for uninformed investors. 
Proof. See the appendix.

Proposition 1 extends Vives (1995), restating a result due to He and Wang (1995), providing an alternative, constructive proof. According to (13), at any period $n$ the equilibrium price is a weighted average of the market expectation about the fundamentals $v$, and a monotone transformation of the $n$-th period aggregate demand intercept. ${ }^{22}$ A straightforward rearrangement of (13) yields

$$
\begin{aligned}
p_{n}-E_{n}[v] & =\frac{\alpha_{P_{n}}}{a_{n}} E_{n}\left[\theta_{n}\right] \\
& =\Lambda_{n}\left(a_{n}\left(v-E_{n}[v]\right)+\theta_{n}\right) .
\end{aligned}
$$

According to (16), the discrepancy between $p_{n}$ and $E_{n}[v]$ is due to the contribution that noise traders are expected to give to the $n$-th period aggregate demand. The parameter $\Lambda_{n} \equiv \alpha_{P_{n}} / a_{n}$ is a measure of market depth. The smaller is $\Lambda_{n}$ and the smaller is the anticipated (and realized) contribution that the stock of noise gives to the aggregate demand and to the price.

At any period $n<3$, an investor's strategy is the sum of two components. The first component captures the investor's activity based on his private estimation of the difference between the fundamentals and the $n$-th period equilibrium price. This can be considered as "long-term" speculative trading, aimed at profiting from the liquidation value of the asset. The second component captures the investor's activity based on the extraction of order flow, i.e. public, information. This trading is instead aimed at exploiting short-run movements in the asset price determined by the evolution of the future aggregate demand. Upon observing this information, and depending on the sign of the difference $\alpha_{P_{n}}-\alpha_{E_{n}}$, investors engage either in "market making" (when $\alpha_{P_{n}}-\alpha_{E_{n}}<0$, thereby accommodating the aggregate demand) or in "trend chasing" (when $\alpha_{P_{n}}-\alpha_{E_{n}}>0$, thus following the market). ${ }^{23}$

To fix ideas, consider the following example. Suppose that $p_{n}-E_{n}[v]>0$. According to (16), we know that the market attributes the discrepancy between the price and the public expectation to the presence of a positive expected stock of demand coming from noise traders: $E_{n}\left[\theta_{n}\right]>0$. An investor's reaction to this observation depends on whether he believes it to be driven by noise or information. In the former (latter) case, the forward looking attitude implied by rational behavior, would advise the investor to accommodate (join) the aggregate demand in the expectation of a future price reversion (further increase). ${ }^{24}$ Suppose $\alpha_{P_{n}}<\alpha_{E_{n}}$, then informed investors count on the reversal of noise traders' demand in the next period(s) and take the other side of the market, acting as market makers. They thus short the asset expecting to buy it back in the future at a lower price. ${ }^{25}$ If, on the other hand, $\alpha_{P_{n}}>\alpha_{E_{n}}$,

\footnotetext{
${ }^{22}$ This is immediate since in any linear equilibrium $\int_{0}^{1} x_{i n} d i+\theta_{n}=a_{n} v+\theta_{n}-\varphi_{n}\left(p^{n}\right)$.

${ }^{23} \mathrm{He}$ and Wang (1995) point out that in a market with long term investors the weights that prices and average expectations assign to fundamentals can differ.

${ }^{24}$ In other words, owing to the traditional signal extraction problem, it is entirely possible that the sign of $E_{n}\left[\theta_{n}\right]$ is due to the presence of a positive demand coming from informed traders.

${ }^{25}$ When $\alpha_{P_{n}}-\alpha_{E_{n}}<0$, the reaction to the aggregate demand investors display in the above example is akin to a "contrarian" strategy. While value investors tend to buy at low prices in the expectation that the intrinsic value of an asset will eventually show up, our investors take the other side of the market just to exploit the regularity in the pattern of noise traders' demand.
} 
informed investors anticipate that the role of "positive" fundamental information looms large in the $n$-th period aggregate demand and that this is most likely affecting the sign of $E_{n}\left[\theta_{n}\right]$. As a consequence, they buy the asset, expecting to re sell it once its price has incorporated the positive news, effectively chasing the trend. ${ }^{26}$

Finally, note that according to (15), in the third period investors concentrate in "long term speculation." Indeed, at $n=3$, investors anticipate that the asset will be liquidated in the next period and thus that its value will not depend on the information contained in that period's aggregate demand. As a consequence, they choose their position only taking into account their information on the fundamentals, acting like in a static market.

Remark 3. While for $N=3$ existence is daunting to show, assuming $N=2$ we are able to prove that an equilibrium in linear strategies always exists. ${ }^{27}$ In this latter case, multiple equilibria may in principle arise. For some parameter values, it is easy to find equilibria. For instance, if noise increments are i.i.d., and investors only receive private information in the first period (i.e., if $\beta=1$ and $\left.\tau_{\epsilon_{2}}=0\right)$, there always exists an equilibrium where $a_{1}=a_{2}=(1+\kappa)^{-1} \gamma \tau_{\epsilon_{1}}$, whereas for large values of $\tau_{\delta}$ another equilibrium where $a_{1}=\left(\gamma \tau_{u}\right)^{-1}\left(1+\kappa+\gamma^{2} \tau_{\epsilon_{1}} \tau_{u}\right)>a_{2}=(1+\kappa)^{-1} \gamma \tau_{\epsilon_{1}}$ may also arise (in line with what happens in a model where investors receive a signal containing a common error term - see Remark 1). The first equilibrium disappears when $\beta<1$. In the absence of residual uncertainty (i.e., if $\tau_{\delta}^{-1}=0$ ), $\kappa=0$, and the equilibrium with $a_{1}=a_{2}=\gamma \tau_{\epsilon_{1}}$ is unique (see Section 3.3).

As argued above, the difference $\alpha_{P_{n}}-\alpha_{E_{n}}$ plays a crucial role in shaping investors' reactions to public information and thus their trading behavior. In our static benchmark, on the other hand, the same difference also determines how "close" the price is to the fundamentals compared to the average expectations investors hold about it. This fact is also true in a dynamic market. Indeed, since

$$
\bar{E}_{n}[v] \equiv \int_{0}^{1} E_{i n}[v] d i=\alpha_{E_{n}} v+\left(1-\alpha_{E_{n}}\right) E_{n}[v],
$$

and using (13), a straightforward extension of the argument used in section 2 allows to obtain the following

Corollary 1. At any linear equilibrium of the 3-period market, the following three conditions are equivalent:

$$
\begin{aligned}
\left|E\left[p_{n}-v \mid v\right]\right| & >\left|E\left[\bar{E}_{n}[v]-v \mid v\right]\right| \\
\alpha_{P_{n}} & <\alpha_{E_{n}} \\
\operatorname{Cov}\left[p_{n}, v\right] & <\operatorname{Cov}\left[\bar{E}_{n}[v], v\right] .
\end{aligned}
$$

Proof. To prove the equivalence between (17) and (18), we use here the direct proof, based on the analysis of the market clearing equation, adopted in Section 2. Using the expression for

\footnotetext{
${ }^{26}$ Note that the intensity of the trading based on order flow information is positively related to the depth of the period $n$ market. Indeed, in a deeper market both a market maker and a market chaser face smaller adverse price movements, and are thus willing to trade more aggressively.

${ }^{27}$ The proof is available from the authors upon request.
} 
strategies in Proposition 1, at any period $n<3$ at equilibrium we have

$$
\int_{0}^{1} x_{i n} d i+\theta_{n}=0 \Leftrightarrow \frac{a_{n}}{\alpha_{E_{n}}}\left(\bar{E}_{n}[v]-p_{n}\right)+\frac{\alpha_{P_{n}}-\alpha_{E_{n}}}{\alpha_{E_{n}}} \frac{a_{n}}{\alpha_{P_{n}}}\left(p_{n}-E_{n}[v]\right)+\theta_{n}=0 .
$$

Solving for the price and rearranging yields

$$
p_{n}=\bar{E}_{n}[v]+\frac{\alpha_{P_{n}}-\alpha_{E_{n}}}{a_{n}} E_{n}\left[\theta_{n}\right]+\frac{\alpha_{E_{n}}}{a_{n}} \theta_{n},
$$

where $E_{n}\left[\theta_{n}\right]=a_{v}\left(v-E_{n}[v]\right)+\theta_{n}$. This, in turn, implies that

$$
p_{n}-v=\bar{E}_{n}[v]-v+\frac{\alpha_{P_{n}}-\alpha_{E_{n}}}{a_{n}} E_{n}\left[\theta_{n}\right]+\frac{\alpha_{E_{n}}}{a_{n}} \theta_{n} .
$$

Thus, if $\alpha_{P_{n}}>\alpha_{E_{n}}$ the price is closer to the fundamentals compared the consensus opinion, while the opposite occurs whenever $\alpha_{P_{n}}<\alpha_{E_{n}}$.

For the second part of the proof, computing the covariance between $p_{n}$ and $v$ yields

$$
\operatorname{Cov}\left[v, p_{n}\right]=\alpha_{P_{n}} \frac{1}{\tau_{v}}+\left(1-\alpha_{P_{n}}\right)\left(\frac{1}{\tau_{v}}-\frac{1}{\tau_{n}}\right),
$$

and carrying out a similar computation for the time $n$ consensus opinion

$$
\operatorname{Cov}\left[\bar{E}_{n}[v], v\right]=\alpha_{E_{n}} \frac{1}{\tau_{v}}+\left(1-\alpha_{E_{n}}\right)\left(\frac{1}{\tau_{v}}-\frac{1}{\tau_{n}}\right)
$$

where $\tau_{n} \equiv \operatorname{Var}\left[v \mid p^{n}\right]=\tau_{v}+\tau_{u} \sum_{t=1}^{n} \Delta a_{t}^{2}$. We can now subtract (21) from (20) and obtain

$$
\operatorname{Cov}\left[p_{n}-\bar{E}_{n}[v], v\right]=\frac{\alpha_{P_{n}}-\alpha_{E_{n}}}{\tau_{n}},
$$

implying that the price at time $n$ over relies on public information if and only if the covariance between the price and the fundamentals falls short of that between the consensus opinion and the fundamentals.

We can now put together the results obtained in proposition 1 and corollary 1: if upon observing the $n$-th period aggregate demand investors expect it to be mostly driven by noise trades, they accommodate the order flow. As a consequence, their behavior drives the price away from the fundamentals compared to the average market opinion. If, instead, they deem the aggregate demand to be mostly information driven, they align their short term positions to those of the market. This, in turn, drives the price closer to the fundamentals, compared to investors' average expectations.

Alternatively, when investors speculate on short term returns the equilibrium price and the consensus opinion have different dynamics:

$$
p_{n}=\bar{E}_{n}[v]+\frac{\alpha_{P_{n}}-\alpha_{E_{n}}}{a_{n}} E_{n}\left[\theta_{n}\right]+\frac{\alpha_{E_{n}}}{a_{n}} \theta_{n} .
$$

Indeed, as the price originates from market clearing, it reflects both determinants of investors' demand, i.e. their long term forecast and their short term speculative activity. Conversely, as 
the consensus opinion is only based on investors' long term expectations, it does not reflect the impact of short term speculation.

To establish the direction of inequality (17) we thus need to determine what is the force that drives an investor's reaction to the information contained in the aggregate demand. Prior to that we consider a special case of our model in which investors do not receive private signals at any period $n$. In this case short term speculation is disconnected from the existence of overor under-reliance of prices on public information, as we show in the following section.

\subsection{Homogeneous Information and Short Term Speculation}

In this section we assume away heterogeneous information, setting $\tau_{\epsilon_{n}}=0$, for all $n$. This considerably simplifies the analysis and allows us to show that in the absence of heterogeneous information short term speculation does not lead prices to be systematically closer or farther away from the fundamentals compared to investors' average expectations. We start by characterizing the equilibrium in this setup, and then analyze its properties.

Proposition 2. In the 3-period market with homogeneous information, there exists a unique equilibrium in linear strategies, where prices are given by

$$
p_{n}=\bar{v}+\Lambda_{n} \theta_{n}
$$

where

$$
\begin{gathered}
\Lambda_{3}=\frac{1+\kappa}{\gamma \tau_{v}} \\
\Lambda_{2}=\Lambda_{3}\left(1+\frac{(\beta-1) \gamma^{2} \tau_{u} \tau_{v}}{1+\kappa+\gamma^{2} \tau_{u} \tau_{v}}\right) \\
\Lambda_{1}=\Lambda_{2}\left(1+\frac{(\beta-1) \gamma^{2} \tau_{u} \tau_{v}\left((1+\kappa)(1-\beta)+\gamma^{2} \tau_{u} \tau_{v}\right)}{\left(1+\kappa+\gamma^{2} \beta^{2} \tau_{u} \tau_{v}\right)(1+\kappa)+\gamma^{2} \tau_{u} \tau_{v}\left(1+\kappa+\gamma^{2} \tau_{u} \tau_{v}\right)}\right)
\end{gathered}
$$

and $\kappa=\tau_{v} / \tau_{\delta}$. Risk averse speculators trade according to

$$
X_{n}\left(p^{n}\right)=-\Lambda_{n}^{-1}\left(p_{n}-\bar{v}\right), \quad n=1,2,3 .
$$

Proof. See the appendix

In a market with homogeneous information, at any period $n$ investors have no private signal to use when forming their position. As a consequence, the aggregate demand only reflects the stock of noise trades. According to (28), this implies that speculators always take the other side of the market, buying the asset when $p_{n}<\bar{v} \Leftrightarrow \theta_{n}=\Lambda_{n}^{-1}\left(p_{n}-\bar{v}\right)<0$, and selling it otherwise. Indeed, in the absence of private information, risk averse investors face no adverse selection problem when they clear the market. The discrepancy between the equilibrium price and the unconditional expected value reflects the risk premium investors demand in order to accommodate the liquidity needs of noise traders. Even in the absence of adverse selection risk, in fact, investors anticipate the possibility that the liquidation value $v$ may be lower (higher) than the price they pay for (at which they sell) the asset. 
If $\beta<1$, risk averse investors also speculate on short term asset price movements providing additional order flow accommodation at any time $n=1,2$. This can be seen rearranging (28) in the following way:

$$
X_{n}\left(p^{n}\right)=\Lambda_{3}^{-1}\left(\bar{v}-p_{n}\right)-\left(\Lambda_{n}^{-1}-\Lambda_{3}^{-1}\right)\left(p_{n}-\bar{v}\right) .
$$

As a result, for $\beta \in(0,1)$, market depth decreases across trading periods:

$$
0<\Lambda_{1}<\Lambda_{2}<\Lambda_{3}
$$

and within each period it decreases in $\beta$ :

$$
\frac{\partial \Lambda_{n}}{\partial \beta}>0
$$

as one can immediately see from (25), (26), and (27). The intuition for these results is that if $\beta<1$, as noise trades increments are negatively correlated, prior to the last trading round investors have more opportunities to unload their risky position. This reduces the risk they bear, and lowers the impact that the noise shock has on the price. If $\beta=1$ noise trades increments are i.i.d.. Therefore, speculators cannot count on the future reversion in the demand of noise traders and their extra order flow accommodation disappears. As a consequence, depth is constant across periods: $\Lambda_{1}=\Lambda_{2}=\Lambda_{3}=\left(\gamma \tau_{v}\right)^{-1}(1+\kappa) .{ }^{28}$

As one would intuitively expect, short term speculation arises insofar as investors can map the partial predictability of noise trades' increments into the anticipation of short term returns. The following proposition formalizes this intuition:

Corollary 2. At $n=1,2$ investors speculate on short term asset price movements if and only if, provided $\theta_{n}>0\left(\theta_{n}<0\right)$, they expect the next period return to revert: $E_{n}\left[p_{n+1}-p_{n}\right]<0$ $\left(E_{n}\left[p_{n+1}-p_{n}\right]>0\right)$.

Proof. Using (24) we can easily obtain

$$
E_{n}\left[p_{n+1}-p_{n}\right]=\left(\beta \Lambda_{n+1}-\Lambda_{n}\right) \theta_{n}
$$

Fixing $n=2$, and using (26) we then obtain

$$
\begin{aligned}
\left(\beta \Lambda_{3}-\Lambda_{2}\right) \theta_{2} & =\Lambda_{3}(\beta-1) \frac{1+\kappa}{1+\kappa+\gamma^{2} \tau_{u} \tau_{v}} \theta_{2} \\
& =\Lambda_{3} \Lambda_{2}^{-1}(\beta-1) \frac{1+\kappa}{1+\kappa+\gamma^{2} \tau_{u} \tau_{v}}\left(p_{2}-\bar{v}\right)
\end{aligned}
$$

In a similar way, fixing $n=1$, and using (27) yields

$$
\begin{aligned}
\left(\beta \Lambda_{2}-\Lambda_{1}\right) \theta_{1} & =\Lambda_{2}(\beta-1) \frac{\left(1+\kappa+\gamma^{2} \beta^{2} \tau_{u} \tau_{v}\right)(1+\kappa)+\gamma^{2} \beta(1+\kappa) \tau_{u} \tau_{v}}{\left(1+\kappa+\gamma^{2} \tau_{u} \tau_{v}\right)(1+\kappa)+\gamma^{2} \tau_{u} \tau_{v}\left(1+\kappa+\gamma^{2} \tau_{u} \tau_{v}\right)} \theta_{1} \\
& =\Lambda_{2} \Lambda_{1}^{-1} \frac{(\beta-1)\left(1+\kappa+\gamma^{2} \beta^{2} \tau_{u} \tau_{v}\right)(1+\kappa)+\gamma^{2} \beta(1+\kappa) \tau_{u} \tau_{v}}{\left(1+\kappa+\gamma^{2} \tau_{u} \tau_{v}\right)(1+\kappa)+\gamma^{2} \tau_{u} \tau_{v}\left(1+\kappa+\gamma^{2} \tau_{u} \tau_{v}\right)}\left(p_{1}-\bar{v}\right)
\end{aligned}
$$

\footnotetext{
${ }^{28}$ This matches the result that He and Wang obtain when looking at the case of homogeneous information when signal are fully informative on $v$, i.e. with $\tau_{\epsilon_{n}} \rightarrow \infty$.
} 
Since for $\beta \in[0,1)$, the terms multiplying $\theta_{n}$ in (29) and (30) are both negative, $E_{n}\left[p_{n+1}-p_{n}\right]<$ $0 \Leftrightarrow \theta_{n}>0$. If $\beta=1$ investors do not speculate on short term returns, and $\Lambda_{1}=\Lambda_{2}=\Lambda_{3}=$ $\left(\gamma \tau_{v}\right)^{-1}(1+\kappa)$. This, in turn, implies that $E_{n}\left[p_{n+1}-p_{n}\right]=0$, for $n=1,2$, proving our claim.

Both in the market with homogeneous information and in the one with heterogeneous information investors speculate on short term returns. However, while in the latter market this possibly leads to the fact that prices over-rely on public information, in the presence of symmetric information this never happens:

Corollary 3. With homogeneous information at $n=1,2,3$, the price is as far away from the fundamentals as investors' average expectations.

Proof. According to (24), the equilibrium price can be expressed as the sum of investors' average expectations and a noise term $\theta_{n}$ which is by assumption orthogonal to $v$. Hence,

$$
E\left[p_{n}-v \mid v\right]=E\left[\bar{v}+\Lambda_{n} \theta_{n}-v \mid v\right]=\bar{v}-v .
$$

Given that investors do not have private information, the price only reflects the noise term $\theta_{n}$, and $E_{i n}[v]=E[v]=\bar{v}$. Hence,

$$
E\left[\bar{E}_{n}[v]-v \mid v\right]=\bar{v}-v
$$

Thus $E\left[\bar{E}_{n}[v]-v \mid v\right]=E\left[p_{n}-v \mid v\right]$, which proves our result.

As risk-averse investors have no private information to trade with, their orders do not impound fundamental information in the price. As a consequence, as shown in Proposition 2, at any period $n$ investors are able to extract the realization of the noise stock $\theta_{n}$ from the observation of the aggregate demand, implying that the price perfectly reflects $\theta_{n}$. As the latter is orthogonal to $\bar{v}$, and in the absence of heterogeneous signals $\bar{E}_{n}[v]=\bar{v}$, both prices and speculators' consensus opinion about fundamentals stand at the same "distance" from $v$.

The last result of this section draws an implication of our analysis for the time series behavior of returns, showing that second and third period returns display reversal if noise trade increments are correlated:

Corollary 4. At $n=1,2,3$ returns exhibit reversal if and only if $\beta<1$.

Proof. To see this, first we compute the covariance between second and third period returns:

$$
\begin{aligned}
\operatorname{Cov}\left[p_{3}-p_{2}, p_{2}-p_{1}\right] & =\left(\Lambda_{2}\left(\beta \Lambda_{3}-\Lambda_{2}\right)+\beta\left(\beta \Lambda_{3}-\Lambda_{2}\right)\left(\beta \Lambda_{2}-\Lambda_{1}\right)\right) \tau_{u}^{-1} \\
& =\left(\frac{\beta \Lambda_{3}-\Lambda_{2}}{\tau_{u}}\right)\left(\Lambda_{2}\left(1+\beta^{2}\right)-\beta \Lambda_{1}\right)
\end{aligned}
$$

Given that as argued above $\Lambda_{1}<\Lambda_{2}$, a necessary and sufficient condition for $\operatorname{Cov}\left[p_{3}-p_{2}, p_{2}-\right.$ $\left.p_{1}\right]<0$ is that $\left(\beta \Lambda_{3}-\Lambda_{2}\right)<0$. We know from $(29)$ that

$$
\beta \Lambda_{3}-\Lambda_{2}=(\beta-1)(1+\kappa)\left(\frac{1}{\gamma \tau_{v}}+\frac{\gamma \tau_{v}}{1+\kappa+\gamma^{2} \tau_{u} \tau_{v}}\right)<0
$$


for all $\beta \in[0,1)$. Similarly, from (30), $\operatorname{Cov}\left[p_{2}-p_{1}, p_{1}-\bar{v}\right]=\Lambda_{1}\left(\beta \Lambda_{2}-\Lambda_{1}\right) \tau_{u}^{-1}<0$ for $\beta \in[0,1)$. Finally, $\operatorname{Cov}\left[v-p_{3}, p_{3}-p_{2}\right]=-\Lambda_{3}\left(\Lambda_{3}+\beta\left(\beta \Lambda_{3}-\Lambda_{2}\right)\left(1+\beta^{2}\right)\right) \tau_{u}^{-1}<0$ for $\beta \in[0,1)$.

With homogeneous information, reversal occurs because with $\beta<1$, the impact of liquidity shocks "evaporates" across trading periods. Thus, a given liquidity shock $u_{n}$ has a stronger impact on the $n$-th period price compared to the $(n+1)$-th price. As a consequence, the price change spurred by $u_{n}$ across times $n$ and $n+1$ is negative, and more than compensates for any effect generated by the former periods' liquidity shocks, implying that $\operatorname{Cov}\left[p_{n+1}-p_{n}, p_{n}-p_{n-1}\right]<$ 0 . To be sure, consider the following example. Suppose that $u_{1}, u_{2}>0$. Then first period noise traders' demand has a positive impact on the first period price which is larger than the one it has on the second and third period prices. In turn, the second period noise traders' demand has a stronger positive impact on the second period price than on the third period price. Formally: $p_{3}-p_{2}=\Lambda_{3} u_{3}+\beta\left(\beta \Lambda_{3}-\Lambda_{2}\right) u_{2}+\beta u_{1}\left(\beta \Lambda_{3}-\Lambda_{2}\right)$, and $p_{2}-p_{1}=\Lambda_{2} u_{2}+\left(\beta \Lambda_{2}-\Lambda_{1}\right) u_{1}$, with $\beta \Lambda_{n}-\Lambda_{n-1}<0$. Thus, both $u_{1}$ and $u_{2}$ have an impact on $\operatorname{Cov}\left[p_{3}-p_{2}, p_{2}-p_{1}\right]$, the former is positive while the latter is negative. At equilibrium the latter effect is always stronger than the former.

Summarizing, in the model with homogeneous information investors speculate on short term asset price movements if and only if they can exploit the predictability of future noise trades' increments. However, this is not enough to induce over- or under-reliance of prices on public information. Indeed, in the absence of heterogeneous information, prices are as far away from fundamentals as the consensus opinion. Furthermore, corollaries 2 and 4 imply that at any time $n=1,2$, and for all $\left(\beta, 1 / \tau_{\delta}\right) \in[0,1) \times \mathbb{R}_{+}$the short term, contrarian strategy based on the realization of $\theta_{n}$ univocally maps into return reversal.

\subsection{The Effect of Heterogeneous Information}

As explained in Section 3.1, the assumption $\beta<1$ implies that noise trades' increments are negatively correlated, and introduces a mean reverting component in the evolution of the aggregate demand. In the market with homogeneous information analyzed in Section 3.2, as the noise stock is perfectly observable, this leads investors to speculate on short term returns, providing additional order flow accommodation. When investors have private signals, the aggregate demand features also a component that reflects fundamental information. As a consequence, the noise stock cannot be perfectly retrieved, and informed investors face an adverse selection problem. Thus, when faced with the aggregate demand, they estimate the noise stock and choose the side of the market on which to stand, based on which component (noise or information) they trust will influence the evolution of the future aggregate demand. Mean reversion in noise increments pushes investors to take the other side of the market (see Section 3.2). In this section we will argue that with heterogeneous information, if $\tau_{\delta}^{-1}>0$ investors scale up their signal responsiveness prior to the last trading round. This, in turn, implies that prior to the last trading round informed investors are more inclined to attribute a given aggregate demand realization to the impounding of fundamental information, and are pushed to follow the market. Both effects eventually bear on the magnitude of the weight the price assigns to 
the fundamentals:

Proposition 3. In the presence of residual uncertainty, at any linear equilibrium the weight the price assigns to the fundamentals at time $n=1,2$ is given by

$$
\begin{gathered}
\alpha_{P_{1}}=\alpha_{E_{1}}\left(1+\left(\beta \rho_{1}-\rho_{2}\right) \Upsilon_{1}^{1}+\left(\beta \rho_{2}-1\right) \Upsilon_{1}^{2}\right) \\
\alpha_{P_{2}}=\alpha_{E_{2}}\left(1+\left(\beta \rho_{2}-1\right) \Upsilon_{2}^{1}\right),
\end{gathered}
$$

where

$$
\rho_{n}=\frac{a_{n}(1+\kappa)}{\gamma \sum_{t=1}^{n} \tau_{\epsilon_{t}}}
$$

$\kappa=\tau_{\delta}^{-1} \tau_{i 3}$, and the expressions for $\Upsilon_{n}^{k}, a_{n}$ are provided in the appendix for $k, n \in\{1,2\}$ (see equations (61), (79), (80), and (41), (56), (81), respectively). The parameter $\Upsilon_{2}^{1}$ is positive. Numerical simulations show that $\Upsilon_{1}^{1}>0$ and $\Upsilon_{1}^{2}>0$, and that $\rho_{1} \geq \rho_{2} \geq 1{ }^{29}$

Proof. See the appendix.

According to the above result, at any linear equilibrium the magnitude of $\alpha_{P_{n}}$ depends on the sign of the differences $\beta \rho_{1}-\rho_{2}$ and $\beta \rho_{2}-1$. While $\beta<1$ implies that noise traders' demand increments are negatively correlated, $\rho_{n}$ captures the deviation that residual uncertainty induces in investors' signal responsiveness with respect to the "long term" solution. ${ }^{30}$

To better separate the impact that noise traders' mean reversion and the residual uncertainty affecting fundamentals have on $\alpha_{P_{n}}$, we start by considering the case in which $\tau_{\delta}^{-1}=0$. In this case $\kappa=0$, and there exists a unique equilibrium in linear strategies in the market (He and Wang (1995) and Vives (1995)). Furthermore, $\rho_{n}=1$ for all $n$, and a closed form solution is available which partially simplifies the analysis and allows to show

Corollary 5. In the absence of residual uncertainty, at any period $n=1,2$, (a) $a_{n}=\gamma \sum_{t=1}^{n} \tau_{\epsilon_{t}}$, and (b) the $n$-th period price displays over reliance on public information if and only if $\beta<1$.

Proof. See the appendix.

According to the above result, if $\tau_{\delta}^{-1}=0$, investors' responsiveness to private information matches the static solution. Hence, $\rho_{n}=1$ and (31)-(32) become

$$
\begin{gathered}
\alpha_{P_{1}}=\alpha_{E_{1}}\left(1+(\beta-1)\left(\Upsilon_{1}^{1}+\Upsilon_{1}^{2}\right)\right) \\
\alpha_{P_{2}}=\alpha_{E_{2}}\left(1+(\beta-1) \Upsilon_{2}^{1}\right) .
\end{gathered}
$$

We know that $\Upsilon_{2}^{1}>0$ from proposition 3. In the appendix we show that $\tau_{\delta}^{-1}=0$ implies $\Upsilon_{1}^{1}+\Upsilon_{1}^{2}>0$, lending support to part (b) of the above corollary. Intuitively, if $\tau_{\delta}^{-1}=0$, when $\beta<1$ at any time $n=1,2$ the only source of predictability in the future aggregate

\footnotetext{
${ }^{29}$ Simulations have been run assuming that either private information flows at a constant rate in the three trading periods $\left(\tau_{\epsilon_{n}}=\tau_{\epsilon_{1}}\right.$, for $\left.n=2,3\right)$ or that it arrives in the first period only $\left(\tau_{\epsilon_{n}}=0\right.$, for $\left.n=2,3\right)$ with the following parameter values: $\tau_{v}, \tau_{u}, \tau_{\epsilon_{1}} \in\{.1, .2, \ldots, 2\}, \beta \in\{0, .1, \ldots, 1\}$ and $\gamma \in\{1,3\}, \tau_{\delta} \in\{1,10\}$.

${ }^{30}$ If at time $n=1,2$ investors were to neglect short run price movements and be forced to focus on long term speculation only, they would respond to their private information according to $(1+\kappa)^{-1} \gamma \sum_{t=1}^{n} \tau_{\epsilon_{t}}$.
} 
demand comes from the mean reverting nature of the noise trading process, and investors' short term behavior is akin to the one they display in the market with homogeneous information. Thus, upon observing $p_{n}>E_{n}[v] \Leftrightarrow E_{n}\left[\theta_{n}\right]>0\left(p_{n}<E_{n}[v] \Leftrightarrow E_{n}\left[\theta_{n}\right]<0\right)$, investors accommodate the expected positive noise traders' demand (supply), selling (buying) the asset in the anticipation of a future price reversion. As these price movements do not reflect fundamental information, this drives the price away from the terminal pay off.

Corollary 5 argues that, absent residual uncertainty, investors' sole motive to speculate on price differences is the possibility to profit from the mean reversion of noise trades. This suggests that shutting down this prediction channel should eliminate any short term speculative activity:

Corollary 6. In the absence of residual uncertainty and assuming $\beta=1, \alpha_{P_{n}}=\alpha_{E_{n}}$ for $n=1,2$.

Proof. This follows immediately by replacing $\beta=1$ in (34) and (35).

If $\tau_{\delta}^{-1}=0$, and $\beta=1$, noise trades increments are i.i.d. and at any period $n<3$ investors have no way to exploit the predictability of future periods' aggregate demand. As a consequence, they concentrate their trading activity on long term speculation, and $\alpha_{P_{n}}=\alpha_{E_{n}}$.

We can now bring back the effect of residual uncertainty. As argued in section 3.1 in the last trading round agents concentrate on the long term value of the asset, speculating as in a static market without exploiting any pattern in the evolution of the aggregate demand. This implies that their responsiveness to private information is given by

$$
a_{3}=\frac{\gamma \sum_{t=1}^{3} \tau_{\epsilon_{t}}}{1+\kappa}
$$

The above expression generalizes (11) and shows that in a static market with residual uncertainty, the weight investors assign to private information is the risk-tolerance weighted sum of their private signal precisions, scaled down by a factor $1+\kappa$, which is larger, the larger is $\tau_{\delta}^{-1}$. Indeed, the larger is $\tau_{\delta}^{-1}$, the larger is the impact of residual uncertainty on the fundamentals, and the less informative are investors' private signals about the liquidation value. Thus, investors feel less confident about their information and scale down their signal responsiveness.

Residual uncertainty also affects a investor's signal responsiveness at any time $n<3$, and this is reflected by the parameter

$$
\rho_{n}=\frac{a_{n}(1+\kappa)}{\gamma \sum_{t=1}^{n} \tau_{\epsilon_{t}}}
$$

Expression (36), captures the deviation from the long term private signal responsiveness due to the presence of residual uncertainty. As stated in proposition 3 our numerical simulations show that in the presence of residual uncertainty $\rho_{1} \geq \rho_{2} \geq 1$. Thus, prior to the last trading round, investors react to their private signals more aggressively than if they were just about to observe the liquidation value:

$$
a_{n} \geq \frac{\gamma \sum_{t=1}^{n} \tau_{\epsilon_{t}}}{1+\kappa}, n=1,2 .
$$


Indeed, while residual uncertainty makes investors less confident about their signals, the presence of additional trading rounds increases the opportunities to adjust suboptimal positions prior to liquidation. This, in turn, boosts investors' reaction to private information, the more, the longer is the amount of time prior to liquidation, as more trading opportunities are available to revise investors' positions. Furthermore, this also implies that a given aggregate demand realization may be driven by informed investors, contributing to explain the component capturing trading based on order flow information in investors' strategies:

Corollary 7. In the presence of residual uncertainty, at any linear equilibrium the second period price displays over reliance on public information if and only if $\beta \rho_{2}<1$.

Proof. See the appendix.

To fix ideas, suppose $\beta=1$ and assume that at time 2 investors observe $p_{2}>E_{2}[v]$ (i.e., $\left.E_{2}\left[\theta_{2}\right]>0\right)$. Given that the demand of noise traders displays no predictable pattern, a short term position based on shorting the asset in the anticipation of buying it back at a lower price one period ahead is suboptimal. At the same time, the fact that $\rho_{2} \geq 1$ implies that informed investors react more aggressively to their private signal than in a static market. This generates additional informed trading which may be responsible for the observed price realization. Informed investors thus go long in the asset in the anticipation of a further price increase in the coming period. If $\beta<1$, the mean reversion effect of noise trades kicks in and investors' decisions as to the side of the market in which to position themselves needs to trade off this latter pattern against the one driven by fundamental information.

For trend chasing to be optimal in the first period, the impact of the mean reverting component due to noise trades on future prices must be weaker than the effect of informed investors' overreaction to private information in both periods. As $\Upsilon_{1}^{1}>0$ and $\Upsilon_{1}^{2}>0$, inspection of (31) suggests that this depends on the sign of both $\beta \rho_{1}-\rho_{2}$ and $\beta \rho_{2}-1$. Indeed, we have the following numerical result:

Numerical Result. In the presence of residual uncertainty, at any linear equilibrium of the market, if $\beta \rho_{2}>1$, a sufficient condition for $\alpha_{P_{1}}>\alpha_{E_{1}}$ is that $\beta \rho_{1}>\rho_{2}$.

Thus, in the first period having $\beta \rho_{1}>\rho_{2}$ is not enough to ensure that investors are willing to chase the market if $E_{1}\left[\theta_{1}\right]>0$. The intuition is as follows. In the appendix we show that a investor's first period optimal strategy can also be expressed as follows,

$$
X_{1}\left(s_{i 1}, p_{1}\right)=\Gamma_{1}^{1} E_{i 1}\left[p_{2}-p_{1}\right]+\Gamma_{1}^{2} E_{i 1}\left[x_{i 2}\right]+\Gamma_{1}^{3} E_{i 1}\left[x_{i 3}\right]
$$

where expressions for $\Gamma_{1}^{1}, \Gamma_{1}^{2}$, and $\Gamma_{1}^{3}$ are provided in the appendix. Thus, in the first period both the second and third period expected positions impinge on a investor's decision. Suppose $p_{1}>E_{1}[v]$ (i.e., $E_{1}\left[\theta_{1}\right]>0$ ), and $\beta \rho_{1}>\rho_{2}$, but $\beta \rho_{2}<1$. Upon observing a high first period price, given that $\beta \rho_{1}>\rho_{2}>1$, an investor may think to side with the market in the expectation of selling in period 2 once the anticipated further appreciation has realized. This, however, is 
not enough. Indeed, given that $\beta \rho_{2}<1$, upon observing $E_{2}\left[\theta_{2}\right]>0$, (i.e., $p_{2}>E_{2}[v]$ ) investors in the second period, anticipating their third period position, will take the other side of the market. This, in turn, may depress $p_{2}$ and compromise the trend chasing strategy set up in the first period. Thus, if the mean reverting effect of noise trades leading to extra order flow accommodation in the second period is strong enough, even if $\beta \rho_{1}>\rho_{2}$, in the first period investors will take the other side of the market. This, in turn, implies that $\alpha_{P_{1}}<\alpha_{E_{1}}{ }^{31}$

\subsection{Public Information Reliance and Consensus Opinion: Keynes vs. Hayek}

Summarizing the results we obtained in the previous section, the systematic discrepancy between prices and the consensus opinion in the estimation of the fundamentals, depends on the joint impact that noise trades' mean reversion and informed investors' overreaction to private information have on short term speculative activity. According to corollary 5, lacking residual uncertainty, noise trades' mean reversion pushes informed investors to act as market makers. This pulls the price away from the fundamentals compared to the average market opinion. When residual uncertainty is introduced, corollary 7 together with our numerical results imply that the decision to "make" the market or "chase" the trend arises as a solution to the trade off between the strength of noise trades' mean reversion and that of informed investors' overreaction to private information. Finally, when noise trades' increments are i.i.d., corollary 6 and proposition 3 respectively imply that lacking residual uncertainty investors concentrate on long term speculation only, while introducing residual uncertainty they tend to chase the market. This, in turn, leads to a price that is either as far away from, or closer to the fundamentals compared to investors' average opinion. Table 1 summarizes this discussion.

\begin{tabular}{lllll}
\hline & \multicolumn{3}{c}{ Noise trades' persistence } \\
\cline { 3 - 5 } & & $\beta=0$ & $0<\beta<1$ & $\beta=1$ \\
\hline \multirow{2}{*}{ Residual uncertainty } & $\begin{array}{c}\tau_{\delta}^{-1}=0 \\
\tau_{\delta}^{-1}>0\end{array}$ & $\alpha_{P_{n}}<\alpha_{E_{n}}$ & $\alpha_{P_{n}}<\alpha_{E_{n}}$ & $\alpha_{P_{n}}=\alpha_{E_{n}}$ \\
& & $\alpha_{E_{n}} \lessgtr \alpha_{E_{n}}$ & $\alpha_{P_{n}}>\alpha_{E_{n}}$ \\
\hline
\end{tabular}

Table 1: A summary of the results for $n=1,2$.

Our summary suggests that in both periods and for $\tau_{\delta}^{-1} \geq 0$, there must exist a $\beta$ such that $\alpha_{P_{n}}=\alpha_{E_{n}}$, and investors are willing to forgo short term speculation. Numerical simulations confirm this insight as shown in figures 1 and 2 . The figures plot the locus $\Omega_{n} \equiv\left\{\left(\beta, 1 / \tau_{\delta}\right) \in\right.$

\footnotetext{
${ }^{31}$ Notice that in the absence of residual uncertainty, this could not happen. In that case, the only source of predictability comes from noise trades mean reversion. Thus, given that $\beta$ is constant across time, provided $\beta<1$, the condition for price over- or under-reliance on public information does not change in the two trading periods. To be sure, suppose that $\kappa=0$ and that at time $1 E_{1}\left[\theta_{1}\right]>0$. Investors short the asset expecting to buy it back either in period 2 or 3 . If at time $2 E_{2}\left[\theta_{2}\right]>0$, they keep shorting, coherently with what they decided in period 1.
} 


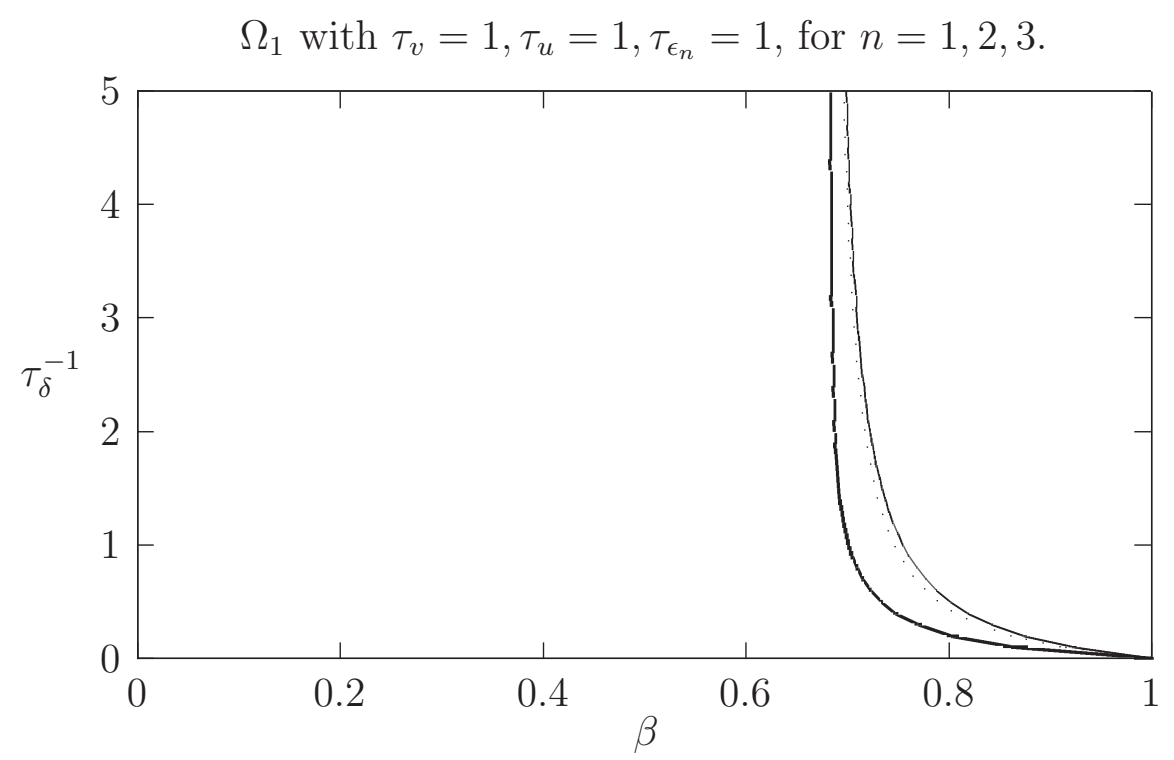

Figure 1: The Keynesian and Hayekian regions for $n=1$ with "constant" arrival of information: $\tau_{\epsilon_{n}}=\tau_{\epsilon}$ for $n=1,2,3$. The bold, dotted, and thin curves are associated respectively to $\gamma=1$, $\gamma=1 / 2$, and $\gamma=1 / 4$. The area to the left of each curve identifies the set of parameter values where over-reliance on public information occurs (i.e., the Keynesian region). Conversely, the area to the right of each curve identifies the set of parameter values yielding under-reliance on public information (the Hayekian region).

$\left.[0,1] \times \mathbb{R}_{+} \mid \alpha_{P_{n}}=\alpha_{E_{n}}\right\}, n=1,2$, assuming that investors receive a private signal in every trading period of the same precision. At any period $n$, the set $\Omega_{n}$ divides the parameter space $\left(\beta, 1 / \tau_{\delta}\right)$ into a Keynesian region (to the left of the locus) with over-reliance on public information, and a Hayekian region (the rest) where the opposite occurs. Formally, the Keynesian region is thus given by the set

$$
\left\{\left(\beta, 1 / \tau_{\delta}\right) \in[0,1] \times \mathbb{R}_{+} \mid \alpha_{P_{n}}<\alpha_{E_{n}}, n=1,2\right\} .
$$

Conversely, the Hayekian region is given by

$$
\left\{\left(\beta, 1 / \tau_{\delta}\right) \in[0,1] \times \mathbb{R}_{+} \mid \alpha_{P_{n}}>\alpha_{E_{n}}, n=1,2\right\} .
$$

With no residual uncertainty $\left(\tau_{\delta}^{-1}=0\right)$ and i.i.d. noise trade increments $(\beta=1), \Omega_{n}=(1,0)$ (corollary 6 ). The introduction of residual uncertainty, on the other hand, may have a nonmonotone effect on $\Omega_{n}$. Observing the figures for small (large) values of $\tau_{\delta}^{-1}$ the Hayekian region widens (shrinks). This is especially true for high levels of risk tolerance. The intuition is as follows. For small levels of residual uncertainty, the fact that speculators can re trade in a dynamic market has a first order impact on $\rho_{n}$ as the possibility to readjust one's position more than compensates for the increase in risk due to the augmented residual uncertainty over the liquidation value. As $\tau_{\delta}^{-1}$ grows larger, the possibility to retrade has an increasingly weaker effect on an investor's dynamic responsiveness, as private signals become less and less relevant to forecast the fundamentals. Investors thus scale back their responsiveness and more noise trades persistence is needed to make investors forgo short term speculation. ${ }^{32}$

\footnotetext{
${ }^{32}$ According to the figures above as $\tau_{\delta}^{-1}$ grows unboundedly investors' private signal responsiveness shrinks
} 


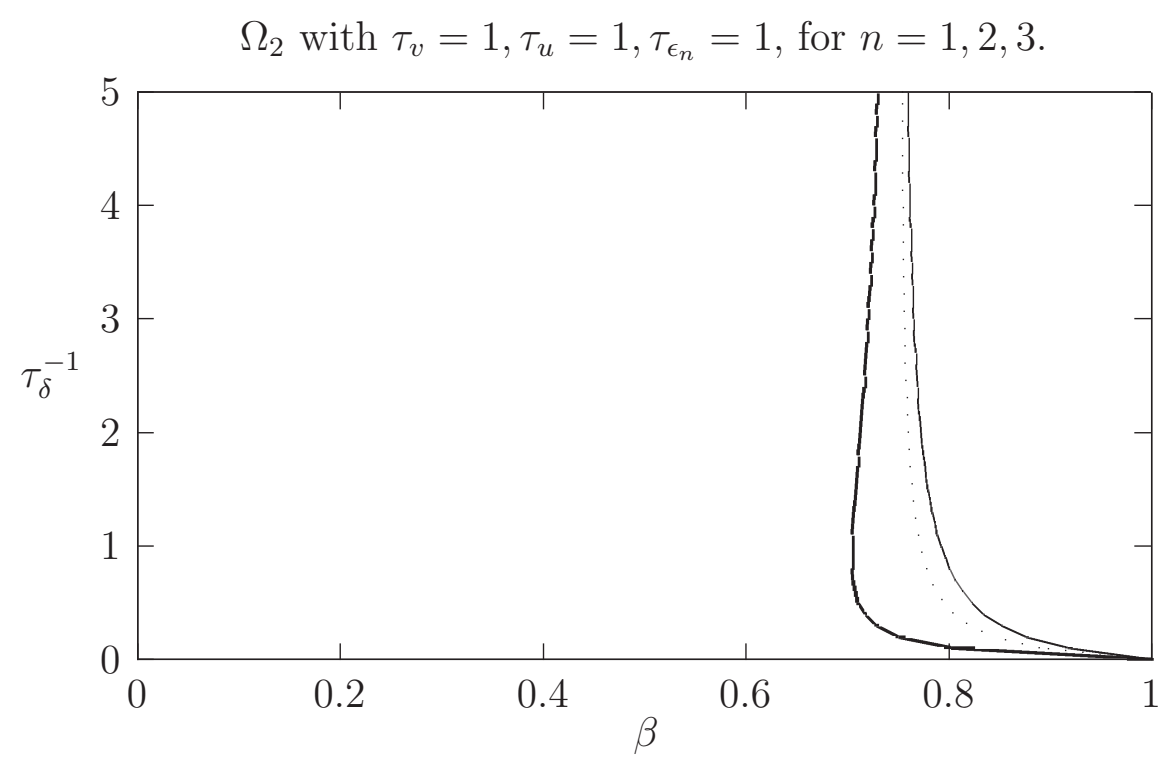

Figure 2: The Keynesian and Hayekian regions for $n=2$ with "constant" arrival of information: $\tau_{\epsilon_{n}}=\tau_{\epsilon}$ for $n=1,2,3$. The bold, dotted, and thin curves are associated respectively to $\gamma=1$, $\gamma=1 / 2$, and $\gamma=1 / 4$. The area to the left of each curve identifies the set of parameter values where over-reliance on public information occurs (i.e., the Keynesian region). Conversely, the area to the right of each curve identifies the set of parameter values yielding under-reliance on public information (the Hayekian region).

According to our simulations, at any trading period the Hayekian (Keynesian) region widens (shrinks) whenever the impact of investors' overreaction to private information on aggregate demand realizations is strong. This occurs for large values of $\gamma, \tau_{\epsilon}$, and $\tau_{u}$. When, on the other hand, $\tau_{v}$ is large, investors enter the market with sufficiently good prior information, and the trading process is unlikely to have a strong informational impact on the price. In this case, the Hayekian (Keynesian) region shrinks (widens). Interestingly, when investors only receive information in the first and second period we find that $\alpha_{P_{2}}<\alpha_{E_{2}}$. Similarly, our numerical simulations show that if $\tau_{\epsilon_{2}}=0$, the same happens in the first period as well, implying that the Hayekian region disappears in both period 1 and 2 , and $\Omega_{n}=\left\{\left(1, \tau_{\delta}^{-1}\right)\right.$, for $\left.\tau_{\delta}^{-1}>0\right\}$. The intuition is as follows: from our previous analysis the reason why informed investors may want to side with the market is that they believe that fundamental information drives the aggregate demand realization. However, with this pattern of information arrival, investors do not receive any new signal after the first (or second) trading round. As a consequence, in the presence of a mean reverting demand from noise traders, siding with the market exposes informed investors to a considerable risk of trading in the expectation of a price increase (decrease) in the second and third period and instead being faced with a price decrease (increase). ${ }^{33}$

but the Hayekian region does not disappear. In the 2-period model it is easy to see that when $\tau_{\delta}^{-1} \rightarrow \infty$, $\Omega_{1}$ becomes a constant. Indeed, in this case $\Omega_{1}=\left\{\left(\beta, 1 / \tau_{\delta}\right) \in[0,1] \times \mathbb{R} \mid \beta \rho_{1}=1\right\}$, and $\lim _{\tau_{\delta}^{-1} \rightarrow \infty} \rho_{1}=$ $\left(\tau_{v}+\tau_{\epsilon_{1}}\right)^{-1}\left(\tau_{v}+\tau_{\epsilon_{1}}+\tau_{\epsilon_{2}}\right)>1$ is a constant that only depends on deep parameters. Therefore, $\beta \rho_{1}=1$ can be explicitly solved, yielding $\beta=\left(\tau_{v}+\tau_{\epsilon_{1}}+\tau_{\epsilon_{2}}\right)^{-1}\left(\tau_{v}+\tau_{\epsilon_{1}}\right)<1$. In the three-period model our numerical simulations show that a similar effect is at work.

${ }^{33}$ The figures in the text refer to a set of numerical simulations that were conducted assuming $\tau_{v}, \tau_{u}, \tau_{\epsilon_{n}} \in$ $\{1,4\}, \gamma \in\{1 / 4,1 / 2,1\}$, and $\beta \in\{0,0.001,0.002, \ldots, 1\}, \tau_{\delta}^{-1} \in\{0.1,0.2, \ldots, 5\}$, for each pattern of private information arrival. 
The set $\Omega_{n}$ captures the space of deep parameter values granting the existence of an equilibrium in which investors only focus on an asset "long-term prospects and those only." This is the attitude towards investment that Keynes contrasted to the Beauty Contest (General Theory, Ch. 12). The exclusive focus on an asset long term prospects arises either in the absence of any systematic pattern in the evolution of the aggregate demand (as argued in corollary 6) or when the forces backing trend chasing are exactly offset by those supporting market making (as shown in figures 1 and 2). In both cases, along the region $\Omega_{n}$, long term investors can only devote their attention to forecasting the fundamentals, shying away from the exploitation of the profits generated by short-term price movements. As a consequence, the price ends up being as close to the fundamentals as the market average opinion.

Corollary 2 argues that in the presence of symmetric information it is possible to map observed price departures from the public expectation at a given period $n$ (i.e., $p_{n}-E_{n}[v]$ ), into a position which is coherent with investors' expectations about the future evolution of the market price. The following corollary shows that an equivalent result also holds in the market with heterogeneous information, characterizing the consensus opinion about the evolution of future prices in the Hayekian and Keynesian regions:

Corollary 8. In the presence of residual uncertainty, at any linear equilibrium

$$
E\left[p_{2}-E_{2}[v] \mid v\right]>0 \Leftrightarrow E\left[\bar{E}_{2}\left[p_{3}-p_{2}\right] \mid v\right]>0
$$

if and only if $\alpha_{P_{2}}>\alpha_{E_{2}}$. If $\tau_{\delta}^{-1}=0$

$$
E\left[p_{n}-E_{n}[v] \mid v\right]>0 \Leftrightarrow E\left[\bar{E}_{n}\left[p_{n+1}-p_{n}\right] \mid v\right]<0 .
$$

Proof. See the appendix.

Thus, in the Hayekian (Keynesian) region, a systematic positive price departure from the public expectation about the fundamentals at time 2 "generates" the consensus opinion that prices will systematically further rise (decrease) in the third period. In the first period numerical simulations confirm that a similar result holds: $E\left[p_{1}-E_{1}[v] \mid v\right]>0 \Leftrightarrow E\left[\bar{E}_{1}\left[p_{2}-p_{1}\right] \mid v\right]>0$. If $\tau_{\delta}^{-1}=0$ informed investors never overreact to their private information. Hence, provided $\beta<1$, only the Keynesian equilibrium can arise and a systematic positive discrepancy between prices and public expectations creates the consensus opinion that prices will systematically revert. Finally, along the region $\Omega_{n}$, the market consensus opinion is that the next period price won't change in any systematic way. As a consequence, $E\left[\bar{E}_{n}\left[p_{n+1}-p_{n}\right] \mid v\right]=0$, and investors concentrate on the asset long term prospects.

\section{Reversal and Momentum}

A vast empirical literature has evidenced the existence of return predictability based on a stock's past performance. DeBondt and Thaler (1986) document a "reversal" effect, whereby stocks with low past returns (losers) tend to outperform stocks with high past returns (winners) over medium/long future horizons. Jegadeesh and Titman (1993), instead, document a 
"momentum" effect, showing that recent past winners tend to outperform recent past losers in the following near future. In our framework, as we argued in Section 3.2, when investors have homogeneous information, noise trades' low persistence implies that returns are negatively correlated, and thus exhibit reversal. ${ }^{34}$

In this section we consider the model with heterogeneous information, and analyze its implications for returns' correlation. The introduction of a strongly persistent factor affecting asset prices (i.e., fundamental information) contrasts the impact of the transient component represented by the noise stock. As a consequence, and except for the case in which $\beta=0$, momentum and reversal can arise in both the Keynesian and the Hayekian equilibrium.

Using (16), we concentrate on the covariance between second and third period returns, as this fully depends on endogenous prices:

$$
\begin{aligned}
\operatorname{Cov}\left[p_{3}-p_{2}, p_{2}-p_{1}\right]=\operatorname{Cov}[ & \left.E_{3}[v]-E_{1}[v], \Lambda_{3} E_{3}\left[\theta_{3}\right]-\Lambda_{1} E_{1}\left[\theta_{1}\right]\right] \\
+ & \operatorname{Cov}\left[\Lambda_{2} E_{2}\left[\theta_{2}\right]-\Lambda_{1} E_{1}\left[\theta_{1}\right], \Lambda_{3} E_{3}\left[\theta_{3}\right]-\Lambda_{2} E_{2}\left[\theta_{2}\right]\right] .
\end{aligned}
$$

Explicitly computing the covariances in (37) and rearranging yields:

$$
\begin{aligned}
\operatorname{Cov}\left[p_{3}-p_{2}, p_{2}-p_{1}\right]= & \left(\frac{\beta \Lambda_{3}-\Lambda_{2}}{\tau_{u}}\right) \times \\
& \left(\Lambda_{2}\left(1+\beta^{2}\right)-\beta \Lambda_{1}+\frac{a_{2} \tau_{u}\left(1-\alpha_{P_{2}}\right)}{\tau_{2}}-\frac{\beta a_{1} \tau_{u}\left(1-\alpha_{P_{1}}\right)}{\tau_{1}}\right) .
\end{aligned}
$$

The latter expression shows that in a market with heterogeneous information the covariance of returns is generated by two effects. The first one is captured by

$$
\left(\frac{\beta \Lambda_{3}-\Lambda_{2}}{\tau_{u}}\right)\left(\Lambda_{2}\left(1+\beta^{2}\right)-\beta \Lambda_{1}\right)
$$

which coincides with the expression given for the third period returns' covariance in the model with homogeneous information. As we argued in Section 3.2, this component reflects the impact of the noise shocks affecting the first and second period aggregate demand. The second component is given by

$$
\left(\frac{\beta \Lambda_{3}-\Lambda_{2}}{\tau_{u}}\right)\left(\frac{a_{2} \tau_{u}\left(1-\alpha_{P_{2}}\right)}{\tau_{2}}-\frac{\beta a_{1} \tau_{u}\left(1-\alpha_{P_{1}}\right)}{\tau_{1}}\right),
$$

and captures the impact of the fundamental information shocks affecting the first and second period aggregate demand.

Inspection of (38) shows that if $\beta=0$, then $\operatorname{Cov}\left[p_{3}-p_{2}, p_{2}-p_{1}\right]<0$, implying that if noise trades' increments are strongly negatively correlated (i.e., the stock of noise trades is transient,

\footnotetext{
${ }^{34}$ More in detail, DeBondt and Thaler (1986) classify all the NYSE-traded stocks according to their past three-year return in relation to the corresponding market average in the period spanning January 1926 to December 1982 in stocks that outperform the market ("winners") and stocks that underperform it ("losers"). According to their results, in the following three years, portfolios of losers outperform the market by $19.6 \%$ on average while portfolios of winners underperform the market by $5 \%$ on average. Jegadeesh and Titman (1993), classify NYSE stocks over the period from January 1963 to December 1989 according to their past six-month returns. Their results show that the top prior winners tend to outperform the worst prior losers by an average of $10 \%$ on an annual basis. Research on momentum and reversal is extensive (see Vayanos and Woolley (2008) and Asness, Moskowitz and Pedersen (2008) for a survey of recent contributions).
} 
and i.i.d), returns can only exhibit reversal. Hence, when $\beta=0$ equilibria are Keynesian (in that the price over relies on public information) and display negative returns' autocorrelation.

As $\beta$ increases away from zero, depending on the patterns of private information arrival, momentum can arise. To see this, we start by assuming away residual uncertainty and set $\beta=1$, so that any pattern in the correlation of returns must depend on the time distribution of private information. In this situation, as argued in Corollary 6 , the equilibrium is unique and we have $\alpha_{P_{n}}=\alpha_{E_{n}}=\tau_{\text {in }}^{-1} \sum_{t=1}^{n} \tau_{\epsilon_{t}}, a_{n}=\gamma \sum_{t=1}^{n} \tau_{\epsilon_{t}}$, and

$$
\Lambda_{n}=\frac{1}{\gamma \tau_{\text {in }}}
$$

implying that, provided investors receive information at all trading dates, and differently from what happens in the market with homogeneous information, market depth improves over time. ${ }^{35}$ As a consequence, $\Lambda_{3}<\Lambda_{2}$ and, similarly to the case with homogeneous information, the impact of a given liquidity shock "evaporates" across trading periods. Note, however, that as now market depth depends on the patterns of information arrival, the presence of heterogeneous information makes it possible for the impact of the first period liquidity shock to overpower that of the liquidity shock arriving in the second period. Indeed, as one can verify:

$$
\begin{aligned}
\operatorname{Cov}\left[p_{3}-p_{2}, p_{2}-p_{1}\right]>0 & \Leftrightarrow 2 \Lambda_{2}-\Lambda_{1}+\frac{a_{2} \tau_{u}\left(1-\alpha_{P_{2}}\right)}{\tau_{2}}-\frac{a_{1} \tau_{u}\left(1-\alpha_{P_{1}}\right)}{\tau_{1}}<0 \\
& \Leftrightarrow \tau_{\epsilon_{2}}>\frac{\tau_{i 1}}{1+\gamma \tau_{u} a_{1}},
\end{aligned}
$$

and given that $\left(1+\gamma \tau_{u} a_{1}\right)^{-1} \tau_{i 1}>\tau_{\epsilon_{1}}$, we can conclude that with no residual uncertainty and i.i.d. noise increments, returns are positively correlated provided that investors receive private information at all trading dates (i.e., $\tau_{\epsilon_{n}}>0$, for all $n$ ), and the quality of such information shows sufficient improvement across periods 1 and 2 . In this situation, market depth considerably increases between the first and second period. This implies that the impact of the first period liquidity shock is always stronger than the one coming from $u_{2}$, building a positive trend in returns. ${ }^{36}$ Furthermore, a large second period private precision strengthens the impact of fundamental information, eventually yielding $\operatorname{Cov}\left[p_{3}-p_{2}, p_{2}-p_{1}\right]>0$.

When $\beta<1$ (keeping $\tau_{\delta}^{-1}=0$ ), noise trades' persistence is lower and this helps to generate a negative covariance. As a result, the value of $\tau_{\epsilon_{2}}$ which is needed for the model to display momentum, increases. Adding residual uncertainty, lowers investors' responsiveness to private information. This, in turn, implies that for any $\beta$, the value of $\tau_{\epsilon_{2}}$ that triggers momentum further increases (see Figure 3).

Summarizing, when $\beta=0$ as argued in section 3.4 the Keynesian equilibrium realizes. There we obtain excessive reliance on public information, and prices that are farther away from the fundamentals compared to the consensus opinion. Investors accommodate a positive expected liquidity demand, as the consensus opinion is that prices systematically revert. Furthermore, returns are negatively correlated. As $\beta$ grows larger, for intermediate values of the residual

\footnotetext{
${ }^{35}$ In the market with homogeneous information if $\beta=1, \Lambda_{n}=\left(\gamma \tau_{v}\right)^{-1}(1+\kappa)$, for $n=1,2,3$.

${ }^{36}$ Formally, $2 \Lambda_{2}-\left.\Lambda_{1}\right|_{\tau_{\epsilon_{2}}=\left(1+\gamma a_{1} \tau_{u}\right)^{-1} \tau_{i 1}}<0$.
} 


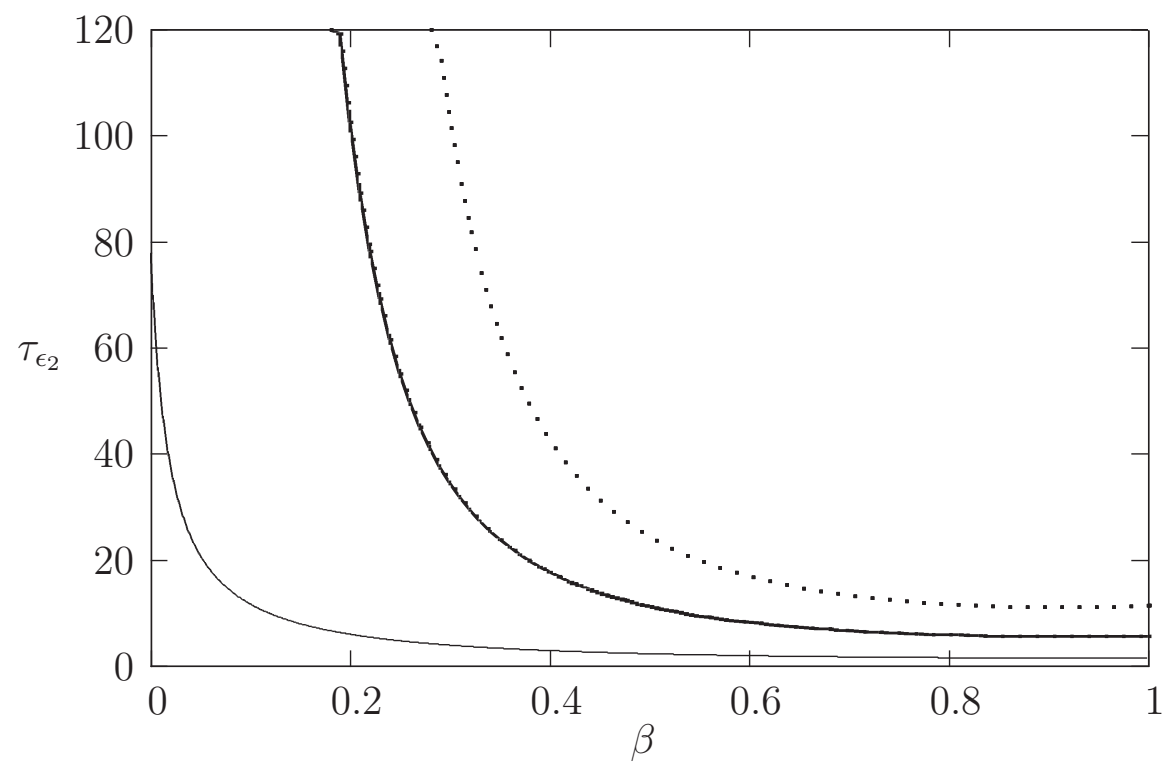

Figure 3: The figure displays the set $\left\{\left(\beta, \tau_{\epsilon_{2}}\right) \in[0,1] \times \mathbb{R}_{+} \mid \operatorname{Cov}\left[p_{3}-p_{2}, p_{2}-p_{1}\right]=0\right\}$, partitioning the parameter space $[0,1] \times \mathbb{R}_{+}$into two regions: points above the plot identify the values of $\left(\beta, \tau_{\epsilon_{2}}\right)$ such that there is momentum. Points below the plot identify the values of $\left(\beta, \tau_{\epsilon_{2}}\right)$ such that there is reversal. Parameters' values are $\tau_{v}=\tau_{u}=\tau_{\epsilon_{1}}=\tau_{\epsilon_{3}}=1$. The thin, thick and dotted line respectively correspond to $\tau_{\delta}^{-1}=0, \tau_{\delta}^{-1}=.2$, and $\tau_{\delta}^{-1}=.3$.

uncertainty parameter the Hayekian equilibrium may occur, with insufficient reliance on public information, and prices that are closer to the fundamentals compared to the consensus opinion. Upon observing a positive realization of the expected liquidity demand, investors chase the trend, as in this case the consensus opinion is that prices will systematically increase. In this equilibrium, momentum obtains provided that the quality of investors' private information improves sufficiently across trading dates. Momentum and reversal are therefore compatible with both types of equilibria. ${ }^{37}$

Inspection of figure 3 suggests that for a given $\tau_{\epsilon_{2}}$, higher values of $1 / \tau_{\delta}$ require a larger noise trades' persistence for $\operatorname{Cov}\left[p_{3}-p_{2}, p_{2}-p_{1}\right]=0$. Numerical simulations confirm this insight, showing that the set of parameter values $\left(\beta, 1 / \tau_{\delta}\right)$ for which $\operatorname{Cov}\left[p_{3}-p_{2}, p_{2}-p_{1}\right]$ is null has the shape displayed by the thick line in figure 4 . Points above (below) the thick line represent combinations of $\left(\beta, 1 / \tau_{\delta}\right)$ such that the third period returns display reversal (momentum), so that $\operatorname{Cov}\left[p_{3}-p_{2}, p_{2}-p_{1}\right]<0\left(\operatorname{Cov}\left[p_{3}-p_{2}, p_{2}-p_{1}\right]>0\right)$. It is useful to also draw the set $\Omega_{2}=\left\{\left(\beta, 1 / \tau_{\delta}\right) \in[0,1] \times \mathbb{R}_{+} \mid \alpha_{P_{2}}=\alpha_{E_{2}}\right\}$ for the chosen parameter configuration. This partitions the parameter space $[0,1] \times \mathbb{R}_{+}$into four regions. Starting from the region $H R$ in which there is under reliance on public information and reversal and moving clockwise, we have the region $H M$ with under reliance on public information and momentum; the region $K M$ with over reliance on public information and momentum; the region $K R$ with over reliance on public information and reversal. ${ }^{38}$

\footnotetext{
${ }^{37}$ Therefore, as momentum can arise also in the Keynesian region, a price runup is entirely compatible with a situation in which prices are farther away from the fundamentals compared with the consensus opinion.

${ }^{38}$ In the figure we use parameters' values in line with Cho and Krishnan (2000)'s estimates based on S\&P500 data. Thus, we set $\tau_{v}=1 / 25, \tau_{u}=1 / 0.011^{2}, \gamma=1 / 2$ and $\tau_{\epsilon_{1}}=1 / 144, \tau_{\epsilon_{2}}=\tau_{\epsilon_{3}}=4 / 144$.
} 


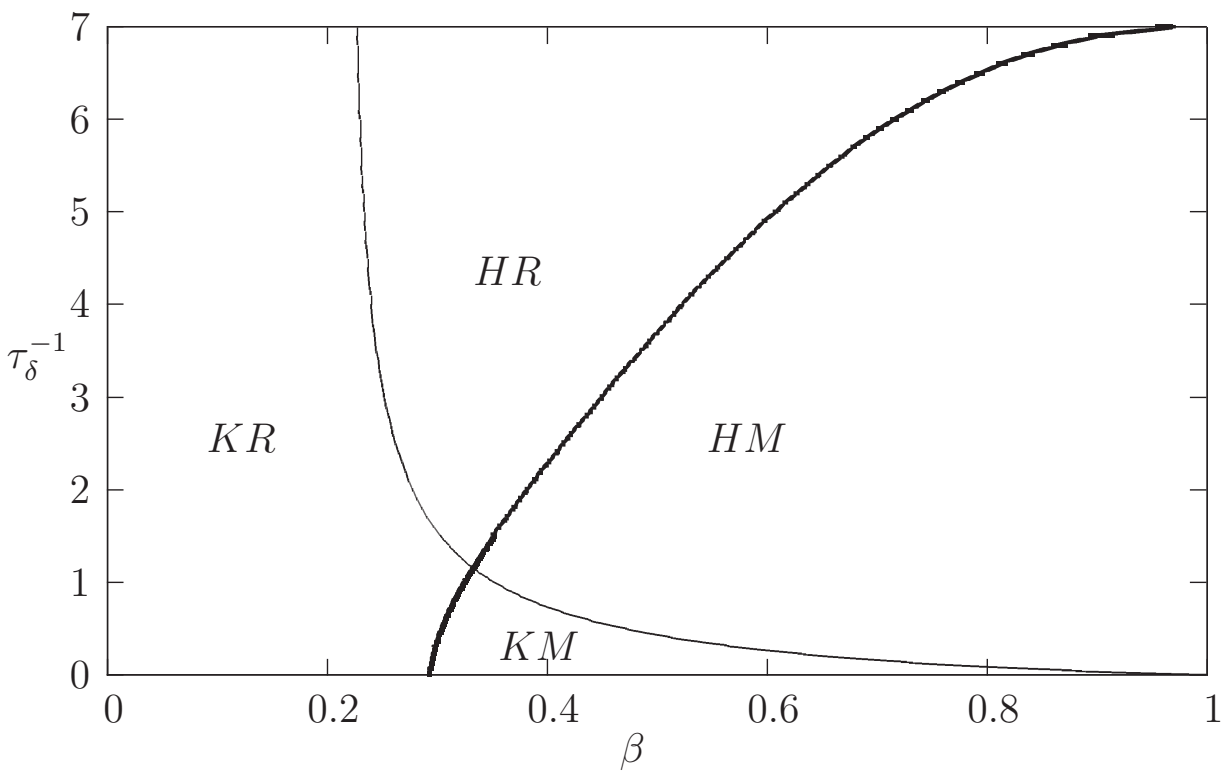

Figure 4: The figure displays the set $\Omega_{2}=\left\{\left(\beta, 1 / \tau_{\delta}\right) \in[0,1] \times \mathbb{R}_{+} \mid \alpha_{P_{2}}=\alpha_{E_{2}}\right\}$ (thin line) and the set $\left\{\left(\beta, 1 / \tau_{\delta}\right) \in[0,1] \times \mathbb{R}_{+} \mid \operatorname{Cov}\left[p_{3}-p_{2}, p_{2}-p_{1}\right]=0\right\}$ (thick line). Parameters' values are $\tau_{v}=1 / 25, \tau_{u}=1 / 0.011^{2}, \gamma=1 / 2$ and $\tau_{\epsilon_{1}}=1 / 144, \tau_{\epsilon_{2}}=\tau_{\epsilon_{3}}=4 / 144$.

According to Corollary 8, in the Hayekian (Keynesian) region investors' short term strategies reflect the consensus opinion about the systematic behavior of future prices. For instance, in the region to the right of $\Omega_{2}$ (i.e., the region $H$ ), a systematic positive discrepancy between $p_{2}$ and $E_{2}[v]$ creates the consensus opinion that the third period price will increase above $p_{2}$. This rationalizes informed investors' decisions to ride the market upon observing $p_{2}-$ $E_{2}[v]>0$. As figure 4 clarifies, in this region the consensus opinion about the systematic future price behavior does not always coincide with the forecast based on unconditional correlation. Indeed, suppose that at time 2 investors observe $p_{2}>p_{1}>E_{2}[v]$. For $\left(\beta, 1 / \tau_{\delta}\right) \in H R$, unconditional correlation predicts that the short term increase in prices across the first two periods will be followed by a reversal, in stark contrast with the prediction based on the consensus opinion. To understand the reason for this difference, it is useful to refer to the case with homogeneous information. In that case, upon observing the realization of a positive noise stock $\theta_{2}>0$, investors speculate on short run price differences by taking the other side of the market. Furthermore, unconditional correlation predicts a price reversal. Indeed, with homogeneous information the only factor moving prices is represented by noise traders' demand which is transient. Therefore, both a positive liquidity stock and a price increase are deemed to be temporary. In the presence of heterogeneous information, on the other hand, fundamental information, which is persistent, also affects prices. This contrasts the mean reverting impact of noise, creating a signal extraction problem, and implying that investors have to base their short term strategies on the realization of the expected noise stock, $E_{2}\left[\theta_{2}\right]$, filtered out of the observed aggregate demand. In this situation, it is natural that the anticipation of future price behavior crucially depends on the information set on which such a forecast is based.

The latter result is reminiscent of Biais, Bossaerts, and Spatt (2008) who study the empirical implications that a multi-asset, dynamic, noisy rational expectations equilibrium model has for 
optimal trading behavior. One of their findings points to the existence of a discrepancy between momentum strategies based on unconditional correlation and the optimal, price contingent strategies that investors adopt in their model. ${ }^{39}$

\section{Conclusions}

In this paper we have investigated the relationship between prices and consensus opinion as estimators of the fundamentals. We have shown that whenever heterogeneously informed, long term investors find it optimal to exploit short term price movements, prices can either be systematically farther away or closer to the fundamentals compared to the consensus opinion. This gives rise to a Keynesian and a Hayekian region in the space of our deep parameters (i.e., the persistence of noise trades and the dispersion of residual uncertainty affecting the asset liquidation value). In the Hayekian (Keynesian) region a systematic positive price departure from the public expectation about the fundamentals "generates" the consensus opinion that prices will systematically further rise (decrease) in the upcoming period. On the boundary between the two regions, on the other hand, the market consensus opinion is that the next period price won't change in any systematic way. As a consequence, investors concentrate on "the asset long term prospects and those only," abiding by Keynes's dictum.

Our paper provides a number of empirical implications. According to our results, for a given level of residual uncertainty, investors tend to use accommodating strategies when noise trading is strongly mean reverting. Conversely, they are trend chasers when noise trading is close to random walk and there is a continuous flow of private information. The latter parameter region widens when investors are more risk tolerant, receive better private information and a lower level of noise affects prices.

Furthermore, as in our setup the evolution of prices is governed by a transient and a persistent component, depending on the quality of private information, our model can generate empirically documented return regularities. Interacting the space of parameter values yielding momentum and reversal with the Keynesian and Hayekian regions, we have illustrated that the set of deep parameters yielding the two phenomena are different. As we argued, the consensus opinion can be taken as a measure of the market view of an asset fundamentals which, differently from the market price, is free from the influence of short term speculation dynamics. Therefore, our theory gives indications as to when a price runup (momentum) should be associated with a situation in which prices are a better or worse indicator of the liquidation value compared to consensus. Low residual uncertainty in the liquidation value together with a high noise trades' persistence are likely to characterize situations of the first type. On the other hand, low noise trades' persistence (again coupled with low residual uncertainty) can be responsible of prices growing increasingly apart from fundamentals compared to the market consensus opinion. ${ }^{40}$

\footnotetext{
${ }^{39}$ Biais, Bossaerts, and Spatt (2008) also find that price contingent strategies are empirically superior to momentum strategies.

${ }^{40}$ From an empirical point of view, our "Hayekian" and "Keynesian" regions can potentially be identified expost by estimating the covariance of prices and consensus with the fundamentals. This enables to characterize when situations in which the market view is at odds with prices are a signal that consensus should be trusted
} 
Overall, our analysis points to the fact that the predictability of the aggregate demand evolution leads long-term investors to speculate on short-term returns, in turn implying that the simplistic EMH is likely to fail. We identify two factors which may explain this result: the persistence of noise trades and the opaqueness of fundamentals. Indeed, as we have shown, low noise trades persistence together with opaque fundamentals make the evolution of the aggregate demand, and thus of the asset returns, predictable. This lures investors towards the exploitation of these regularities, partially diverting them from the activity of evaluating the fundamentals. As a result, the equilibrium price ends up reflecting both components of investors' strategies (long and short term speculation), decoupling its dynamic from that of the consensus opinion. In these conditions, we have also argued that reversal occurs, and prices display over-reliance on public information. Momentum, instead, needs high noise trading persistence, and a transparent environment to arise. Hence, insofar as a high $\beta$ proxies for a high trading frequency, we can conclude that any technological arrangement conducive to an increase in trading frequency together with improved disclosure is likely to promote positive return correlation and price under-reliance on public information.

A number of issues are left for future research. Our analysis has concentrated on the case in which investors have long horizons. Indeed, short term speculation in our setup arises endogenously whenever investors find it optimal to exploit regularities in the evolution of future returns. In a companion paper we analyze the implications of forcing on investors a short term horizon and show that in our general framework this is conducive to multiple equilibria with either Keynesian or Hayekian features (Cespa and Vives (2009)). Furthermore, while our paper gives a very detailed characterization of the conditions leading to investors' over-reliance on public information, it does not assess the welfare consequences that this may have for market participants. In particular, in the Keynesian equilibrium informed investors explicitly take advantage of noise traders, exploiting the low persistence of their demand shocks. A model in which the noise in the price is due to rational traders entering the market to hedge a shock to their endowment would allow to analyze the welfare properties of this equilibrium. Furthermore, it would also allow to see whether in response to informed investors' activity liquidity patterns can change over time, thereby inducing a time-varying degree of noise trades' persistence, and ultimately affecting the sign and magnitude of the discrepancy between prices and average expectations in the estimation of fundamentals. ${ }^{41}$

as a better indicator of ex-post liquidation value. Indeed, as we show in section 4, the fact that momentum and reversal can occur in both the $\mathrm{H}$ and $\mathrm{K}$ regions implies that in some cases we should trust price runups to be strong indicators of value (compared to consensus), whereas in other cases, this is not true. Of course, the testability of these implications relies on the availability of reliable information on consensus estimates which is not easy to obtain because of incentive issues of market professionals which are likely to induce biases (see Vissing-Jorgensen (2003) and the references cited therein). More recently, however, survey data based on investor beliefs which circumvent incentive issues start being collected (see, e.g., Vissing-Jorgensen (2003) and Piazzesi and Schneider (2009)).

${ }^{41}$ Several authors have made a foray into the welfare analysis of noisy, dynamic rational expectations equilibrium models (see, e.g., Brennan and Cao (1996), and Cespa and Foucault (2008)). 


\section{References}

Admati, A. R. (1985). A noisy rational expectations equilibrium for multi-asset securities markets. Econometrica 53(3), 629-657.

Allen, F., S. Morris, and H. S. Shin (2006). Beauty contests and iterated expectations in asset markets. Review of Financial Studies 19(3), 719-752.

Angeletos, G. M. and A. Pavan (2007). Efficiency and welfare in economies with incomplete information. Econometrica 75(4), 1103-1142.

Asness, C. S., T. J. Moskowitz, and L. H. Pedersen (2009). Value and Momentum Everywhere. Working Paper.

Bacchetta, P. and E. van Wincoop (2006a). Can Information Heterogeneity Explain the Exchange Rate Determination Puzzle? American Economic Review 96(3), 552-576.

Bacchetta, P. and E. van Wincoop (2006b). Higher order expectations in asset pricing. Journal of Money, Credit and Banking 40(5), 837-866.

Banerjee, S., Kaniel, R. and I. Kremer (2009). Price Drift as an Outcome of Differences in Higher Order Beliefs. Review of Financial Studies 22(9), 3707-3734.

Bhattacharya, U. and M. Spiegel (1991). Insiders, outsiders, and market breakdowns. Review of Financial Studies 4 (2), 255-282.

Bernstein, P. L. (1998). Against the Gods. The Remarkable Story of Risk. John Wiley \& Sons. New York.

Biais, B. and P. Bossaerts (1998, April). Asset prices and trading volume in a beauty contest. Review of Economic Studies 65(2), 307-340.

Biais, B., P. Bossaerts, and C. Spatt (2008). Equilibrium Asset Pricing and Portfolio Choice Under Asymmetric Information. Review of Financial Studies. Forthcoming.

Brown, D. P. and R. H. Jennings (1989). On technical analysis. Review of Financial Studies $2(4), 527-551$.

Cao, H. H. and H. Ou-Yang (2005, October). Bubbles and panics in a frictionless market with heterogeneous expectations. Working Paper.

Cespa, G. (2002). Short-term investment and equilibrium multiplicity. European Economic Review 46(9), 1645-1670.

Cespa, G. and X. Vives (2009). Higher order expectations and short-term trading. Working Paper.

Cespa, G. and T. Foucault (2008). Insiders-outsiders, transparency, and the value of the ticker. CEPR Discussion Paper \#6794.

Cho, J. and M. Krishnan (2000). Prices as Aggregators of Private Information: Evidence from S\&P 500 Futures Data. Journal of Financial and Quantitative Analysis 35(1), 111-126. 
DeBondt, W. and R. Thaler (1986). Does the stock market overreact? Journal of Finance $40(3), 793-807$.

De Long, B., Shleifer, A., Summers, L. H. and R. J. Waldmann (1990). Noise trader risk in financial markets. Journal of Political Economy 98(4), 703-738.

Easley, D., Engle, R. F., O'Hara, M. and L. Wu (2008). Time-Varying Arrival Rates of Informed and Uninformed Trades. Journal of Financial Econometrics 6(2), 171-207.

Ganguli, J. V. and L. Yang (2009) Complementarities, multiplicity, and supply information. Journal of the European Economic Association 7, 90-115.

Gennotte, G. and H. Leland (1990). Market liquidity, hedging, and crashes. American Economic Review 80(5), 999-1021.

Grossman, S. J. (1989). The Informational Role of Prices. Cambridge: MIT Press.

Grundy, B. D. and M. McNichols (1989). Trade and the revelation of information through prices and direct disclosure. Review of Financial Studies 2(4), 495-526.

Hayek, F. A. (1945). The use of knowledge in society. American Economic Review 35(4), $519-530$.

He, H. and J. Wang (1995). Differential information and dynamic behavior of stock trading volume. Review of Financial Studies 8(4), 919-972.

Hellwig, M. F. (1980). On the aggregation of information in competitive markets. Journal of Economic Theory 22(3), 477-498.

Jegadeesh, N. and S. Titman (1993). Returns to buying winners and selling losers: implications for stock market efficiency. Journal of Finance 48, 65-91.

Kandel, E. and N. D. Pearson (1995). Differential interpretation of public signals and trade in speculative markets. The Journal of Political Economy 103(4), 831-872.

Kondor, P. (2004). Rational trader risk. Working Paper LSE.

Kyle, A. (1985). Continuous auctions and insider trading. Econometrica 53(6), 1315-1336.

Llorente, G. Michaely R., Saar, G. and J. Wang (2002). Dynamic volume-return relation of individual stocks. Review of Financial Studies 15(4), 1005-1047.

Manzano, C. and X. Vives (2010). Public and private learning from prices, strategic substitutability and complementarity, and equilibrium multiplicity. Working Paper.

Medrano, L. A. and X. Vives (2004). Regulating insider trading when investment matters. Review of Finance 8, 199-277.

Morris, S. and H.S. Shin (2002). The social value of public information. American Economic Review 92, 1521-1534.

Nimark, K. P. (2007). Dynamic higher order expectations. Working paper.

Ottaviani, M and P. N. Sørensen (2010). Aggregation of Information and Beliefs: Asset Pricing Lessons from Prediction Markets. Working paper. 
Piazzesi, M. and M. Schneider (2009). Momentum traders in the housing market: survey evidence and a search model. American Economic Review Papers and Proceedings 99, 406-411.

Singleton, K. J. (1987). Asset prices in a time-series model with disparately informed, competitive traders in New approaches to monetary economics, W. A. Burnett and K. J. Singleton, Eds. Cambridge University Press.

Shiller, R. J. (2005). Irrational Exuberance. Princeton, New Jersey: Princeton University Press.

Vayanos, D. and P. Woolley (2008). An Institutional Theory of Momentum and Reversal. Working Paper.

Vissing-Jorgensen, A. (2003). Perspectives on behavioral finance: does "irrationality" disappear with wealth? Evidence from experience and actions. NBER Macroeconomics Annual, 139-208.

Vives, X. (1995). Short-term investment and the informational efficiency of the market. Review of Financial Studies 8(1), 125-160.

Vives, X. (1997). Learning from others: a welfare analysis. Games and Economic Behavior $20(2), 177-200$.

Vives, X. (2008). Information and Learning in Markets. The Impact of Market Microstructure. Princeton University Press.

Wang, J. (1994). A model of competitive stock trading volume. Journal of Political Economy 102(1), 127-168. 


\section{A Appendix}

The following lemma establishes that working with the sequence $z^{n} \equiv\left\{z_{t}\right\}_{t=1}^{n}$ is equivalent to working with $p^{n} \equiv\left\{p_{t}\right\}_{t=1}^{n}$ :

Lemma 2. In any linear equilibrium the sequence of informational additions $z^{n}$ is observationally equivalent to $p^{n}$.

Proof. Consider a candidate equilibrium in linear strategies $x_{i n}=a_{n} \tilde{s}_{i n}-\varphi_{n}\left(p^{n}\right)$. In the first period imposing market clearing yields $\int_{0}^{1} a_{1} s_{i 1}-\varphi_{1}\left(p_{1}\right) d i+\theta_{1}=a_{1} v-\varphi_{1}\left(p_{1}\right)+\theta_{1}=0$ or, denoting with $z_{1}=a_{1} v+\theta_{1}$ the informational content of the first period order-flow, $z_{1}=\varphi_{1}\left(p_{1}\right)$, where $\varphi_{1}(\cdot)$ is a linear function. Hence, $z_{1}$ and $p_{1}$ are observationally equivalent. Suppose now that $z^{n-1}=\left\{z_{1}, z_{2}, \ldots, z_{n-1}\right\}$ and $p^{n-1}=\left\{p_{1}, p_{2}, \ldots, p_{n-1}\right\}$ are observationally equivalent and consider the $n$-th period market clearing condition: $\int_{0}^{1} X_{n}\left(\tilde{s}_{i n}, p^{n-1}, p_{n}\right) d i+\theta_{n}=0$. Adding and subtracting $\sum_{t=1}^{n-1} \beta^{n-t+1} a_{t} v$, the latter condition can be rewritten as follows:

$$
\sum_{t=1}^{n} z_{t}-\varphi_{n}\left(p^{n}\right)=0,
$$

where $\varphi_{n}(\cdot)$ is a linear function, $z_{t}=\Delta a_{t} v+u_{t}$ denotes the informational content of the $t$-th period order-flow, and $\Delta a_{t}=a_{t}-\beta a_{t-1}$. As by assumption $p^{n-1}$ and $z^{n-1}$ are observationally equivalent, it follows that observing $p_{n}$ is equivalent to observing $z_{n}$.

\section{Proof of Proposition 1}

To prove our argument, we proceed backwards. In the last trading period traders act as in a static model and owing to CARA and normality we have

$$
X_{3}\left(\tilde{s}_{i 3}, z^{3}\right)=\gamma \frac{E_{i 3}[v]-p_{3}}{\operatorname{Var}_{i 3}[v+\delta]},
$$

and

$$
p_{3}=\alpha_{P_{3}}\left(v+\frac{\theta_{3}}{a_{3}}\right)+\left(1-\alpha_{P_{3}}\right) E_{3}[v],
$$

where

$$
\begin{aligned}
a_{3} & =\frac{\gamma \sum_{t=1}^{3} \tau_{\epsilon_{t}}}{1+\kappa}, \\
\alpha_{P_{3}} & =\frac{\sum_{t=1}^{3} \tau_{\epsilon_{t}}}{\tau_{i 3}}
\end{aligned}
$$

$\kappa=\tau_{\delta}^{-1} \tau_{i 3}$. An alternative way of writing the third period equilibrium price is

$$
p_{3}=\lambda_{3} z_{3}+\left(1-\lambda_{3} \Delta a_{3}\right) \hat{p}_{2},
$$

where

$$
\lambda_{3}=\alpha_{P_{3}} \frac{1}{a_{3}}+\left(1-\alpha_{P_{3}}\right) \frac{\Delta a_{3} \tau_{u}}{\tau_{3}},
$$


captures the price impact of the net informational addition contained in the 3rd period aggregate demand, while

$$
\begin{aligned}
\hat{p}_{2} & =\frac{\alpha_{P_{3}} \tau_{3} \beta\left(\sum_{t=1}^{2} \beta^{2-t} z_{t}\right)+\left(1-\alpha_{P_{3}}\right) a_{3} \tau_{2} E_{2}[v]}{\alpha_{P_{3}} \tau_{3} \beta a_{2}+\left(1-\alpha_{P_{3}}\right) a_{3} \tau_{2}} \\
& =\frac{\gamma \tau_{2} E_{2}[v]+\beta(1+\kappa)\left(z_{2}+\beta z_{1}\right)}{\gamma \tau_{2}+\beta a_{2}(1+\kappa)}
\end{aligned}
$$

$z_{n}=\Delta a_{n} v+u_{n}$, and $\Delta a_{n}=a_{n}-\beta a_{n-1}$.

\section{SECOND PERIOD}

Substituting (39) in the second period objective function, a trader in the second period maximizes

$$
E_{i 2}\left[U\left(\pi_{i 2}+\pi_{i 3}\right)\right]=-E_{i 2}\left[\exp \left\{-\frac{1}{\gamma}\left(\left(p_{3}-p_{2}\right) x_{i 2}+\frac{x_{i 3}^{2} \operatorname{Var}_{i 3}[v+\delta]}{2 \gamma}\right)\right\}\right] .
$$

Let $\phi_{i 2}=\left(p_{3}-p_{2}\right) x_{i 2}+x_{i 3}^{2} \operatorname{Var}_{i 3}[v+\delta] /(2 \gamma)$. The term $\phi_{i 2}$ is a quadratic form of the random vector $Z_{2}=\left(x_{i 3}-\mu_{1}, p_{3}-\mu_{2}\right)^{\prime}$, which is normally distributed (conditionally on $\left\{\tilde{s}_{i 2}, z^{2}\right\}$ ) with zero mean and variance covariance matrix

$$
\Sigma_{2}=\left(\begin{array}{cc}
\operatorname{Var}_{i 2}\left[x_{i 3}\right] & \operatorname{Cov}_{i 2}\left[x_{i 3}, p_{3}\right] \\
\operatorname{Cov}_{i 2}\left[x_{i 3}, p_{3}\right] & \operatorname{Var}_{i 2}\left[p_{3}\right]
\end{array}\right)
$$

where

$$
\begin{gathered}
\operatorname{Var}_{i 2}\left[x_{i 3}\right]=\frac{\left(\Delta a_{3}(1+\kappa)-\gamma \tau_{\epsilon_{3}}\right)^{2} \tau_{u}+\tau_{i 2}\left((1+\kappa)^{2}+\gamma^{2} \tau_{u} \tau_{\epsilon_{3}}\right)}{\tau_{i 2} \tau_{u}(1+\kappa)^{2}} \\
\operatorname{Cov}_{i 2}\left[x_{i 3}, p_{3}\right]=\lambda_{3}\left(\frac{\gamma \tau_{\epsilon_{3}} \Delta a_{3} \tau_{u}-(1+\kappa)\left(\tau_{3}+\sum_{t=1}^{2} \tau_{\epsilon_{t}}\right)}{\tau_{i 2} \tau_{u}(1+\kappa)}\right) \\
\operatorname{Var}_{i 2}\left[p_{3}\right]=\lambda_{3}^{2}\left(\frac{\tau_{3}+\sum_{t=1}^{2} \tau_{\epsilon t}}{\tau_{i 2} \tau_{u}}\right)
\end{gathered}
$$

and

$$
\begin{gathered}
\mu_{1} \equiv E_{i 2}\left[x_{i 3}\right]=\frac{a_{3}\left(1-\lambda_{3} \Delta a_{3}\right)}{\alpha_{E_{3}}}\left(E_{i 2}[v]-\hat{p}_{2}\right) \\
\mu_{2} \equiv E_{i 2}\left[p_{3}\right]=\lambda_{3} \Delta a_{3} E_{i 2}[v]+\left(1-\lambda_{3} \Delta a_{3}\right) \hat{p}_{2} .
\end{gathered}
$$

Writing in matrix form:

$$
\phi_{i 2}=c_{2}+b_{2}^{\prime} Z_{2}+Z_{2}^{\prime} A_{2} Z_{2},
$$

where $c_{2}=\left(\mu_{2}-p_{2}\right) x_{i 2}+\mu_{1}^{2} \operatorname{Var}_{i 3}[v+\delta] /(2 \gamma), b_{2}=\left(\mu_{1} \operatorname{Var}_{i 3}[v+\delta] / \gamma, x_{i 2}\right)^{\prime}$, and $A_{2}$ is a $2 \times 2$ matrix with $a_{11}=\operatorname{Var}_{i 3}[v+\delta] /(2 \gamma)$ and the rest zeroes. Using a well-known result from normal theory we can now rewrite the objective function (46) as

$$
\begin{aligned}
& E_{i 2}\left[U\left(\pi_{i 2}+\pi_{i 3}\right)\right]= \\
& \quad-\left|\Sigma_{2}\right|^{-1 / 2}\left|\Sigma_{2}^{-1}+2 / \gamma A_{2}\right|^{-1 / 2} \times \exp \left\{-1 / \gamma\left(c_{2}-\frac{1}{2 \gamma} b_{2}^{\prime}\left(\Sigma_{2}^{-1}+2 / \gamma A_{2}\right)^{-1} b_{2}\right)\right\} .
\end{aligned}
$$


Maximizing the above function with respect to $x_{i 2}$ yields

$$
x_{i 2}=\Gamma_{2}^{1}\left(\mu_{2}-p_{2}\right)+\Gamma_{2}^{2} \mu_{1},
$$

where

$$
\Gamma_{2}^{1}=\frac{\gamma}{h_{2,22}}, \quad \Gamma_{2}^{2}=-\frac{h_{2,21} \operatorname{Var}_{i 3}[v+\delta]}{\gamma h_{2,22}}
$$

and $h_{2, i j}$ denotes the $i j$-th term of the symmetric matrix $H_{2}=\left(\Sigma_{2}^{-1}+2 / \gamma A_{2}\right)^{-1}$ :

$$
\begin{aligned}
& h_{2,12}=-\frac{\lambda_{3} \tau_{i 3}^{2}(1+\kappa)\left(1-\lambda_{3} \gamma \tau_{\epsilon_{3}} /(1+\kappa)\right)}{D_{2} / \gamma^{2}} \\
& h_{2,22}=\frac{\lambda_{3}^{2} \tau_{i 3}\left((1+\kappa)\left(\tau_{3}+\sum_{t=1}^{2} \tau_{\epsilon_{t}}\right)+\tau_{\epsilon_{3}}\right)}{D_{2} / \gamma^{2}},
\end{aligned}
$$

and

$$
\frac{D_{2}}{\gamma^{2}}=\tau_{i 3}\left(\tau_{i 3}\left(\lambda_{3}^{2} \tau_{i 2}+\left(1-\lambda_{3} \Delta a_{3}\right)^{2} \tau_{u}\right)+\tau_{i 2} \tau_{u} \kappa\right) .
$$

Substituting (48) and (49) into (51) and rearranging yields

$$
X_{2}\left(\tilde{s}_{i 2}, z^{2}\right)=\frac{a_{2}}{\alpha_{E_{2}}}\left(E_{i 2}[v]-\hat{p}_{2}\right)-\frac{\gamma}{h_{2,22}}\left(p_{2}-\hat{p}_{2}\right),
$$

where $a_{2}$ denotes the 2 nd period trading aggressiveness:

$$
a_{2}=\frac{\gamma\left(\sum_{t=1}^{2} \tau_{\epsilon_{t}}\right) \tau_{i 3}(1+\kappa)\left(1+\gamma \tau_{u} \Delta a_{3}\right)}{\left(1+\kappa+\gamma \tau_{u} \Delta a_{3}\right)\left(\tau_{\epsilon_{3}}+\left(\tau_{3}+\sum_{t=1}^{2} \tau_{\epsilon_{t}}\right)(1+\kappa)\right)} .
$$

Imposing market clearing yields

$$
\int_{0}^{1} \frac{a_{2}}{\alpha_{E_{2}}}\left(E_{i 2}[v]-\hat{p}_{2}\right) d i-\frac{\gamma}{h_{2,22}}\left(p_{2}-\hat{p}_{2}\right)+\theta_{2}=0,
$$

which after rearranging implies

$$
\frac{\gamma \tau_{2}\left(\beta \rho_{2}-1\right)}{\gamma \tau_{2}+\beta a_{2}(1+\kappa)} E_{2}\left[\theta_{2}\right]=\frac{\gamma}{h_{2,22}}\left(\hat{p}_{2}-p_{2}\right)
$$

where $\rho_{2} \equiv a_{2}(1+\kappa) /\left(\gamma \sum_{t=1}^{2} \tau_{\epsilon_{t}}\right)$. As a consequence, a trader $i$ 's second period strategy can be written as follows:

$$
X_{2}\left(\tilde{s}_{i 2}, z^{2}\right)=\frac{a_{2}}{\alpha_{E_{2}}}\left(E_{i 2}[v]-p_{2}\right)+\frac{\left(\gamma+h_{2,21}\right)\left(\beta \rho_{2}-1\right) \tau_{2}}{\gamma \tau_{i 3}} E_{2}\left[\theta_{2}\right] .
$$

Using (55) we can obtain an expression for the second period equilibrium price that clarifies the role of the impact of expected noise traders' demand. Indeed, imposing market clearing yields

$$
\frac{a_{2}}{\alpha_{E_{2}}}\left(\bar{E}_{2}[v]-p_{2}\right)+\frac{\left(\gamma+h_{2,21}\right)\left(\beta \rho_{2}-1\right) \tau_{2}}{\gamma \tau_{i 3}} E_{2}\left[\theta_{2}\right]+\theta_{2}=0
$$

where $\bar{E}_{2}[v] \equiv \int_{0}^{1} E_{i 2}[v] d i$. Isolating $p_{2}$ and rearranging we obtain

$$
p_{2}=\alpha_{P_{2}}\left(v+\frac{\theta_{2}}{a_{2}}\right)+\left(1-\alpha_{P_{2}}\right) E_{2}[v]
$$


where

$$
\alpha_{P_{2}}=\alpha_{E_{2}}\left(1+\left(\beta \rho_{2}-1\right) \Upsilon_{2}^{1}\right)
$$

denotes the weight that the second period price assigns to $v$, and

$$
\Upsilon_{2}^{1}=\frac{\gamma \tau_{2} \tau_{u}\left(\gamma \tau_{2}+\beta a_{2}(1+\kappa)+\gamma \tau_{i 2} \kappa\right)}{D_{2}}>0
$$

Using (59) and (60) in (58) yields:

$$
X_{2}\left(\tilde{s}_{i 2}, z^{2}\right)=\frac{a_{2}}{\alpha_{E_{2}}}\left(E_{i 2}[v]-p_{2}\right)+\frac{\alpha_{P_{2}}-\alpha_{E_{2}}}{\alpha_{E_{2}}} \frac{a_{2}}{\alpha_{P_{2}}}\left(p_{2}-E_{2}[v]\right) .
$$

Finally, note that in period 2 as well we can obtain a recursive expression for the price that confirms the formula obtained in (43). Indeed, rearranging (59) we obtain

$$
p_{2}=\lambda_{2} z_{2}+\left(1-\lambda_{2} \Delta a_{2}\right) \hat{p}_{1}
$$

where

$$
\lambda_{2}=\alpha_{P_{2}} \frac{1}{a_{2}}+\left(1-\alpha_{P_{2}}\right) \frac{\Delta a_{2} \tau_{u}}{\tau_{2}},
$$

measures the price impact of the new information contained in the second period aggregate demand (since $\left.\int_{0}^{1} x_{i 2} d i+\theta_{2}=a_{2} v+\theta_{2}-\varphi_{2}\left(p_{1}, p_{2}\right)=z_{2}+\beta z_{1}-\varphi_{2}\left(p_{1}, p_{2}\right)\right)$, and

$$
\hat{p}_{1}=\frac{\alpha_{P_{2}} \tau_{2} \beta z_{1}+\left(1-\alpha_{P_{2}}\right) a_{2} \tau_{1} E_{1}[v]}{\alpha_{P_{2}} \tau_{2} \beta a_{1}+\left(1-\alpha_{P_{2}}\right) a_{2} \tau_{1}} .
$$

An alternative expression for $\lambda_{2}$ is as follows:

$$
\begin{aligned}
\lambda_{2}=\underbrace{\frac{1+\kappa+\gamma \tau_{u} \rho_{2} \Delta a_{2}}{\gamma \rho_{2} \tau_{i 2}}}_{\lambda_{2}^{S}}+ \\
\quad \frac{\left(\beta \rho_{2}-1\right)(1+\kappa) \tau_{u}\left(\gamma \tau_{2}+\beta a_{2}(1+\kappa)+\gamma \tau_{i 2} \kappa\right)\left(\tau_{2}-a_{2} \Delta a_{2} \tau_{u}\right)}{\rho_{2} \tau_{i 2} D_{2}},
\end{aligned}
$$

where $\lambda_{2}^{S}$ denotes the "static" measure of the price impact of trade. The above expression thus highlights how noise trade predictability and the presence of residual uncertainty affect the static measure of the price impact of trade.

\section{First Period}

To solve for the first period strategy, we now plug (51) into the argument of the exponential in (50):

$$
\begin{aligned}
& c_{2}-\frac{1}{2 \gamma} b_{2}^{\prime}\left(\Sigma_{2}^{-1}+2 / \gamma A_{2}\right)^{-1} b_{2}=\left(E_{i 2}\left[p_{3}\right]-p_{2}\right) x_{i 2}+\frac{\operatorname{Var}_{i 3}[v+\delta]}{2 \gamma}\left(E_{i 2}\left[x_{i 3}\right]\right)^{2} \\
& -\frac{1}{2 \gamma}\left(\frac{\operatorname{Var}_{i 3}[v+\delta]}{\gamma} E_{i 2}\left[x_{i 3}\right] \quad x_{i 2}\right)\left(\begin{array}{ll}
h_{2,11} & h_{2,12} \\
h_{2,21} & h_{2,22}
\end{array}\right)\left(\begin{array}{c}
\frac{\operatorname{Var}_{i 3}[v+\delta]}{\gamma} E_{i 2}\left[x_{i 3}\right] \\
x_{i 2}
\end{array}\right) .
\end{aligned}
$$


Carrying out the matrix multiplication and simplifying yields

$$
c_{2}-\frac{1}{2 \gamma} b_{2}^{\prime}\left(\Sigma_{2}^{-1}+2 / \gamma A_{2}\right)^{-1} b_{2}=\frac{1}{2 \gamma}\left(h_{2,22} x_{i 2}^{2}+\frac{\gamma^{2}(1+\kappa)^{2} \tau_{i 2} \tau_{u}}{D_{2}}\left(E_{i 2}\left[x_{i 3}\right]\right)^{2}\right),
$$

implying that

$$
\begin{aligned}
E_{i 2}\left[U\left(\pi_{i 2}+\pi_{i 3}\right)\right]=-\left|\Sigma_{2}\right|^{-1 / 2}\left|\Sigma_{2}^{-1}+(2 / \gamma) A_{2}\right|^{-1 / 2} \times \\
\exp \left\{-\frac{1}{2 \gamma^{2}}\left(h_{2,22} x_{i 2}^{2}+\frac{\gamma^{2}(1+\kappa)^{2} \tau_{i 2} \tau_{u}}{D_{2}}\left(E_{i 2}\left[x_{i 3}\right]\right)^{2}\right)\right\} .
\end{aligned}
$$

The first period objective function now reads as follows:

$$
\begin{aligned}
E_{i 1}\left[U\left(\pi_{i 1}+\pi_{i 2}+\pi_{i 3}\right)\right]=-E_{i 1}[\exp \{- & \frac{1}{\gamma}\left(\left(p_{2}-p_{1}\right) x_{i 1}\right. \\
& \left.\left.\left.+\frac{1}{2 \gamma}\left(h_{2,22} x_{i 2}^{2}+\frac{\gamma^{2}(1+\kappa)^{2} \tau_{i 2} \tau_{u}}{D_{2}}\left(E_{i 2}\left[x_{i 3}\right]\right)^{2}\right)\right)\right\}\right] .
\end{aligned}
$$

Note that since

$$
E_{i 2}\left[x_{i 3}\right]=\frac{\gamma \tau_{2}}{1+\kappa}\left(E_{i 2}[v]-E_{2}[v]\right)-\beta E_{i 2}\left[\theta_{2}\right]
$$

we have

$$
E_{i 2}[v]-E_{2}[v]=\frac{(1+\kappa)\left(E_{i 2}\left[x_{i 3}\right]+\beta E_{i 2}\left[\theta_{2}\right]\right)}{\gamma \tau_{2}},
$$

and replacing the latter in the expression for $x_{i 2}$ yields

$$
E_{i 2}\left[x_{i 3}\right]=\frac{x_{i 2}+\left(1-\beta \rho_{2}\right) E_{i 2}\left[\theta_{2}\right]}{\rho_{2}} .
$$

Thus, denoting by $\phi_{i 1}$ the argument of the exponential in (67) we obtain:

$$
\phi_{i 1}=\left(p_{2}-p_{1}\right) x_{i 1}+\frac{1}{2 \gamma}\left(h_{2,22} x_{i 2}^{2}+\frac{\gamma^{2}(1+\kappa)^{2} \tau_{i 2} \tau_{u}}{D_{2}}\left(\frac{x_{i 2}+\left(1-\beta \rho_{2}\right) E_{i 2}\left[\theta_{2}\right]}{\rho_{2}}\right)^{2}\right) .
$$

Finally, as one can verify, letting $\nu_{1}=\alpha_{E_{2}}, \nu_{2}=-\left(\lambda_{2} \tau_{i 2}\right)^{-1}\left(\tau_{2}-a_{2} \Delta a_{2} \tau_{u}\right)$, and $\nu_{3}=1$, we have

$$
\nu_{1} x_{i 2}+\nu_{2} p_{2}+\nu_{3} E_{i 2}\left[\theta_{2}\right]=\frac{1}{\lambda_{2} \tau_{i 2}}\left(\Delta a_{2} \tau_{u} \beta z_{1}-\tau_{1} E_{1}[v]\right) \equiv c\left(z_{1}\right)
$$

implying that

$$
E_{i 2}\left[\theta_{2}\right]=c\left(z_{1}\right)-\alpha_{E_{2}} x_{i 2}+\frac{\tau_{2}-a_{2} \Delta a_{2} \tau_{u}}{\lambda_{2} \tau_{i 2}} p_{2} .
$$

Given a trader's information set at time $1, c\left(z_{1}\right)$ is a constant. Hence, the uncertainty that a trader $i$ faces at time 1 is reflected in $\phi_{i 1}$ through $p_{2}$ and $x_{i 2}$ only:

$$
\begin{aligned}
\phi_{i 1}=\left(p_{2}-\right. & \left.p_{1}\right) x_{i 1}+\frac{1}{2 \gamma}\left(h_{2,22} x_{i 2}^{2}+\frac{\gamma^{2}(1+\kappa)^{2} \tau_{i 2} \tau_{u}}{\rho_{2}^{2} D_{2}} \times\right. \\
& \left.\left(\left(1-\left(1-\beta \rho_{2}\right) \alpha_{E_{2}}\right) x_{i 2}+c\left(z_{1}\right)\left(1-\beta \rho_{2}\right)+\frac{\left(\tau_{2}-a_{2} \Delta a_{2} \tau_{u}\right)\left(1-\beta \rho_{2}\right)}{\lambda_{2} \tau_{i 2}} p_{2}\right)^{2}\right) .
\end{aligned}
$$


The term $\phi_{i 1}$ is a quadratic form of the random vector $Z_{1} \equiv\left(x_{i 2}-\mu_{1}, p_{2}-\mu_{2}\right)$, which is normally distributed conditionally on $\left\{s_{i 1}, z_{1}\right\}$ with mean zero and variance-covariance matrix

$$
\Sigma_{1}=\left(\begin{array}{cc}
\operatorname{Var}_{i 1}\left[x_{i 2}\right] & \operatorname{Cov}_{i 1}\left[x_{i 2}, p_{2}\right] \\
\operatorname{Cov}_{i 1}\left[x_{i 2}, p_{2}\right] & \operatorname{Var}_{i 1}\left[p_{2}\right]
\end{array}\right)
$$

where $\mu_{1} \equiv E_{i 1}\left[x_{i 2}\right]$,

$$
\mu_{1}=\frac{\left(1-\lambda_{2}^{S} \Delta a_{2}\right) a_{2}}{\alpha_{E_{2}}}\left(E_{i 1}[v]-\hat{p}_{1}\right)+\frac{a_{2} \tau_{1}\left(\alpha_{P_{2}}-\alpha_{E_{2}}\right)}{\alpha_{P_{2}} \alpha_{E_{2}} \tau_{2}}\left(\hat{p}_{1}-E_{1}[v]\right),
$$

and $\mu_{2} \equiv E_{i 1}\left[p_{2}\right]$,

$$
\mu_{2}=\lambda_{2} \Delta a_{2} E_{i 1}[v]+\left(1-\lambda_{2} \Delta a_{2}\right) \hat{p}_{1}
$$

while

$$
\begin{gathered}
\operatorname{Var}_{i 1}\left[x_{i 2}\right]=\frac{\left(\Delta a_{2} \sum_{t=1}^{2} \tau_{\epsilon_{t}}-a_{2} \tau_{\epsilon_{2}}\right)^{2} \tau_{u}+\tau_{i 1}\left(\left(\sum_{t=1}^{2} \tau_{\epsilon_{t}}\right)^{2}+a_{2}^{2} \tau_{u} \tau_{\epsilon_{2}}\right)}{\left(\sum_{t=1}^{2} \tau_{\epsilon_{t}}\right)^{2} \tau_{i 1} \tau_{u}} \\
\operatorname{Cov}_{i 1}\left[x_{i 2}, p_{2}\right]=\lambda_{2}\left(\frac{a_{2} \Delta a_{2} \tau_{u} \tau_{\epsilon_{2}}-\left(\tau_{2}+\tau_{\epsilon_{1}}\right)\left(\sum_{t=1}^{2} \tau_{\epsilon_{t}}\right)}{\left(\sum_{t=1}^{2} \tau_{\epsilon_{t}}\right) \tau_{i 1} \tau_{u}}\right) \\
\operatorname{Var}_{i 1}\left[p_{2}\right]=\lambda_{2}^{2}\left(\frac{\tau_{2}+\tau_{\epsilon_{1}}}{\tau_{i 1} \tau_{u}}\right) .
\end{gathered}
$$

Writing in matrix form:

$$
\phi_{i 1}=c_{1}+b_{1}^{\prime} Z_{1}+Z_{1}^{\prime} A_{1} Z_{1},
$$

where

$$
\begin{gathered}
c_{1}=\left(\mu_{2}-p_{1}\right) x_{i 1}+a_{11} \mu_{1}^{2}+a_{22} \mu_{2}^{2}+m_{3} c\left(z_{1}\right)^{2}+2\left(m_{1} \mu_{1} c\left(z_{1}\right)+m_{2} \mu_{2} c\left(z_{1}\right)+a_{12} \mu_{1} \mu_{2}\right), \\
b_{1}=\left(2\left(a_{11} \mu_{1}+a_{12} \mu_{2}+m_{1} c\left(z_{1}\right)\right), 2\left(a_{22} \mu_{2}+a_{12} \mu_{1}+m_{2} c\left(z_{1}\right)\right)+x_{i 1}\right)^{\prime}, \text { and } \\
A_{1}=\left(\begin{array}{cc}
a_{11} & a_{12} \\
a_{12} & a_{22}
\end{array}\right),
\end{gathered}
$$

with

$$
\begin{gathered}
a_{11}=\frac{h_{2,22}}{2 \gamma}+\frac{a_{22}}{\nu_{2}^{2}}\left(\frac{1-\left(1-\beta \rho_{2}\right) \alpha_{E_{2}}}{1-\beta \rho_{2}}\right)^{2} a_{12}=-\frac{a_{22}}{\nu_{2}}\left(\frac{1-\left(1-\beta \rho_{2}\right) \alpha_{E_{2}}}{1-\beta \rho_{2}}\right) \\
a_{22}=\frac{\left(\nu_{2}\left(1-\beta \rho_{2}\right)\right)^{2}}{2 \gamma}\left(\frac{\gamma^{2}(1+\kappa)^{2} \tau_{i 2} \tau_{u}}{\rho_{2}^{2} D_{2}}\right)
\end{gathered}
$$

and

$$
m_{1}=\frac{a_{22}}{\nu_{2}^{2}} \frac{1-\left(1-\beta \rho_{2}\right) \alpha_{E_{2}}}{1-\beta \rho_{2}}, m_{2}=-\frac{a_{22}}{\nu_{2}}, m_{3}=\frac{a_{22}}{\nu_{2}^{2}} .
$$

Along the lines of the second period maximization problem we then obtain

$$
\begin{aligned}
E_{i 1}\left[U \left(\pi_{i 1}+\right.\right. & \left.\left.\pi_{i 2}+\pi_{i 3}\right)\right]= \\
& -\left|\Sigma_{1}\right|^{-1 / 2}\left|\Sigma_{1}^{-1}+2 / \gamma A_{1}\right|^{-1 / 2} \exp \left\{-1 / \gamma\left(c_{1}-\frac{1}{2 \gamma} b_{1}^{\prime}\left(\Sigma_{1}^{-1}+2 / \gamma A_{1}\right)^{-1} b_{1}\right)\right\} .
\end{aligned}
$$


Maximizing (73) with respect to $x_{i 1}$, solving for $x_{i 1}$ and rearranging yields

$$
X_{1}\left(s_{i 1}, p_{1}\right)=\Gamma_{1}^{1} E_{i 1}\left[p_{2}-p_{1}\right]+\Gamma_{1}^{2} E_{i 1}\left[x_{i 2}\right]+\Gamma_{1}^{3} E_{i 1}\left[x_{i 3}\right]
$$

where

$$
\begin{gathered}
\Gamma_{1}^{1}=\frac{\gamma}{h_{1,22}}, \Gamma_{1}^{2}=-\frac{h_{1,12} h_{2,22}}{\gamma h_{1,22}} \\
\Gamma_{1}^{3}=-\frac{\gamma^{2}(1+\kappa)^{2} \tau_{u}\left(\left(\beta \rho_{2}-1\right) \tau_{i 2} \nu_{2} h_{1,22}+\tau_{i 3}\left(1-\lambda_{3} \Delta a_{3}\right) h_{1,12}\right)}{D_{2} h_{1,22}} .
\end{gathered}
$$

and the terms $h_{1, i j}$ denote the $i j$-th elements of the symmetric matrix $H_{1}=\left(\Sigma_{1}^{-1}+2 / \gamma A_{1}\right)^{-1}$ :

$$
\begin{array}{r}
h_{1,22}=\frac{\tau_{i 3}}{\Phi_{1} D_{2} \rho_{2}^{2} \tau_{\epsilon_{2}}}\left((1+\kappa)^{2}\left(\left(\tau_{2}+\tau_{\epsilon_{1}}\right)\left(\kappa \tau_{u}+\lambda_{3}^{2} \tau_{i 3}\right)+\left(1-\lambda_{3} \Delta a_{3}\right)^{2} \tau_{i 3} \tau_{u}\right)\right. \\
\left.+\lambda_{3}^{2} \rho_{2}^{2} \tau_{\epsilon_{2}}\left((1+\kappa)\left(\tau_{3}+\sum_{t=1}^{2} \tau_{\epsilon_{t}}\right)+\tau_{\epsilon_{3}}\right)\right), \\
h_{1,12}=\frac{(1+\kappa)^{2}}{\Phi_{1} D_{2} \rho_{2}^{2} \tau_{\epsilon_{2}} \gamma^{2} \tau_{i 2} \lambda_{2}\left(\sum_{t=1}^{2} \tau_{\epsilon_{t}}\right)}\left(\left(a_{2} \Delta a_{2} \tau_{u} \tau_{\epsilon_{2}}-\left(\tau_{2}+\tau_{\epsilon_{1}}\right)\left(\sum_{t=1}^{2} \tau_{\epsilon_{t}}\right)\right) D_{2}\right. \\
\left.+\left(\tau_{2}-a_{2} \Delta a_{2} \tau_{u}\right)\left(\beta \rho_{2}-1\right) \tau_{u} \tau_{i 3}\left(1-\lambda_{3} \Delta a_{3}\right) \gamma^{2} \tau_{\epsilon_{2}} \sum_{t=1}^{2} \tau_{\epsilon_{t}}\right),
\end{array}
$$

and

$$
\begin{aligned}
\Phi_{1}=\left|\Sigma_{1}\right|^{-1}(1+ & \frac{2 a_{22}}{\gamma}\left(\operatorname{Var}_{i 1}\left[p_{2}\right]+\operatorname{Var}_{i 1}\left[x_{i 2}\right] \frac{\tau_{i 3}^{2}\left(1-\lambda_{3} \Delta a_{3}\right)^{2}}{\nu_{2}^{2} \tau_{i 2}^{2}\left(1-\beta \rho_{2}\right)^{2}}\right. \\
& \left.\left.-2 \frac{\tau_{i 3}\left(1-\lambda_{3} \Delta a_{3}\right)}{\nu_{2} \tau_{i 2}\left(1-\beta \rho_{2}\right)} \operatorname{Cov}_{i 1}\left[p_{2}, x_{i 2}\right]\right)+\frac{h_{2,22} \operatorname{Var}_{i 1}\left[x_{i 2}\right]}{\gamma^{2}}\right)+2 \frac{a_{22} h_{2,22}}{\gamma^{3}} .
\end{aligned}
$$

Substituting (71) and (72) into (74) and imposing market clearing, yields

$$
p_{1}=\alpha_{P_{1}}\left(v+\frac{\theta_{1}}{a_{1}}\right)+\left(1-\alpha_{P_{1}}\right) E_{1}[v]
$$

where

$$
\alpha_{P_{1}}=\alpha_{E_{1}}\left(1+\left(\beta \rho_{1}-\rho_{2}\right) \Upsilon_{1}^{1}+\left(\beta \rho_{2}-1\right) \Upsilon_{1}^{2}\right),
$$

denotes the weight that the first period price assigns to the fundamentals and

$$
\begin{gathered}
\Upsilon_{1}^{1}=\left(\frac{\gamma}{h_{1,22}}-\frac{a_{1}}{\alpha_{E_{1}}}\right) \frac{h_{1,22} \rho_{1} \tau_{1} \alpha_{E_{2}}}{\gamma a_{2}\left(1-\lambda_{2} \Delta a_{2}\right)}, \\
\Upsilon_{1}^{2}=\left(1-\frac{h_{1,22}}{\gamma}\left(\frac{\gamma}{h_{1,22}}-\frac{a_{1}}{\alpha_{E_{1}}}\right)\right) \frac{\gamma \beta \tau_{1} \tau_{u}}{h_{1,22}\left(1-\lambda_{2} \Delta a_{2}\right) D_{2}} \times \\
\left(\frac{\gamma \tau_{i 3} \lambda_{3}(1+\kappa)^{3}\left(1+\gamma \tau_{u} \Delta a_{3}\right) \Phi_{2}}{\rho_{2}^{2} D_{2} \lambda_{2}}-h_{1,12} h_{2,22}\left(\tau_{i 3}\left(1-\lambda_{3} \Delta a_{3}\right)+\tau_{i 2} \kappa\right)\right) \\
-\left(\frac{\gamma}{h_{1,22}}-\frac{a_{1}}{\alpha_{E_{1}}}\right) \frac{h_{1,22} \rho_{1} \tau_{1} \sum_{t=1}^{2} \tau_{\epsilon_{t}}}{\gamma a_{2} \tau_{2}\left(1-\lambda_{2} \Delta a_{2}\right)} \frac{\tau_{2} \tau_{u}\left(a_{2}(1+\kappa)+\gamma \tau_{2} \beta \rho_{1}\right)\left(\tau_{i 3}\left(1-\lambda_{3} \Delta a_{3}\right)+\tau_{i 2} \kappa\right)}{\tau_{i 2} D_{2}},
\end{gathered}
$$


and $a_{1}$ denotes a trader $i$ 's first period private signal responsiveness:

$$
\begin{aligned}
a_{1}=\frac{\alpha_{E_{1}}}{h_{1,22}}\left(\lambda _ { 2 } \Delta a _ { 2 } \left(\gamma-2\left(h_{1,22} a_{22}+\right.\right.\right. & \left.\left.h_{1,12} a_{12}\right)\right)+ \\
& \left.-2\left(h_{1,22} a_{12}+h_{1,12} a_{11}\right)\left(\frac{a_{2} \tau_{1}}{\sum_{t=1}^{2} \tau_{\epsilon_{t}}}+\beta a_{1}\right)\right) .
\end{aligned}
$$

Using (77) in (74) and rearranging yields

$$
X_{1}\left(s_{i 1}, z_{1}\right)=\frac{a_{1}}{\alpha_{E_{1}}}\left(E_{i 1}[v]-p_{1}\right)+\frac{\alpha_{P_{1}}-\alpha_{E_{1}}}{\alpha_{E_{1}}} \frac{a_{1}}{\alpha_{P_{1}}}\left(p_{1}-E_{1}[v]\right) .
$$

Note that (82), together with (77) show that the expressions for equilibrium prices and traders' strategies have a recursive structure. Finally, note that as obtained in periods 2 and 3, we can express the first period equilibrium price as follows

$$
p_{1}=\lambda_{1} z_{1}+\left(1-\lambda_{1} a_{1}\right) \bar{v}
$$

where

This completes our proof.

$$
\lambda_{1}=\alpha_{P_{1}} \frac{1}{a_{1}}+\left(1-\alpha_{P_{1}}\right) \frac{a_{1} \tau_{u}}{\tau_{1}}
$$

QED

\section{Proof of Proposition 2}

Suppose that $\tau_{\epsilon_{1}}=\tau_{\epsilon_{2}}=\tau_{\epsilon_{3}}=0$. Then, since in equilibrium

$$
\begin{gathered}
a_{3}=\frac{\gamma \sum_{t=1}^{3} \tau_{\epsilon_{t}}}{1+\kappa} \\
a_{2}=\frac{\gamma\left(\sum_{t=1}^{2} \tau_{\epsilon_{t}}\right) \tau_{i 3}(1+\kappa)\left(1+\gamma \tau_{u} \Delta a_{3}\right)}{\left(1+\kappa+\gamma \tau_{u} \Delta a_{3}\right)\left(\tau_{\epsilon_{3}}+\left(\tau_{3}+\sum_{t=1}^{2} \tau_{\epsilon_{t}}\right)(1+\kappa)\right)},
\end{gathered}
$$

we immediately obtain $a_{2}=a_{3}=0$. Note that this is in line with what one should assume in a linear equilibrium where traders possess no private information. Indeed, at any candidate linear equilibrium a trader's strategy at time $n$ is given by $X_{n}\left(p^{n}\right)=\varphi\left(p^{n}\right)$, where $\varphi(\cdot)$ is a linear function. Imposing market clearing, in turn implies that $\varphi\left(p^{n}\right)=\theta_{n}$, so that at any linear equilibrium the price only incorporates the supply shock $\left(a_{n}=0\right)$ which is therefore perfectly revealed to risk averse speculators.

This, in turn, implies that $\tau_{n}=\tau_{\text {in }}=\tau_{v}$,

$$
E_{n}[v]=E_{i n}[v]=\bar{v}
$$

and that $\alpha_{P_{n}}=\alpha_{E_{n}}=0$. Now, we can go on and characterize the strategies that traders adopt, using the expressions that appear in proposition 1 in the paper:

$$
\begin{gathered}
X_{3}\left(p^{3}\right)=\frac{\gamma \tau_{v}}{1+\kappa}\left(\bar{v}-p_{3}\right) \\
X_{2}\left(p^{2}\right)=\frac{\gamma \tau_{v}}{1+\kappa}\left(\bar{v}-p_{2}\right)+\frac{(\beta-1) \gamma^{3} \tau_{v}^{2} \tau_{u}}{(1+\kappa)\left(1+\kappa+\beta \gamma^{2} \tau_{u} \tau_{v}\right)}\left(p_{2}-\bar{v}\right) .
\end{gathered}
$$


The second component of the latter expression, in particular, comes from the fact that

$$
\lim _{\tau_{\epsilon_{n}} \rightarrow 0} \frac{\alpha_{P_{1}}-\alpha_{E_{1}}}{\alpha_{E_{1}}} \frac{a_{1}}{\alpha_{P_{1}}}=\frac{(\beta-1) \gamma^{3} \tau_{v}^{2} \tau_{u}}{(1+\kappa)\left(1+\kappa+\beta \gamma^{2} \tau_{u} \tau_{v}\right)} .
$$

Imposing market clearing we obtain:

$$
p_{2}=\bar{v}+\frac{(\beta-1)(1+\kappa) \gamma \tau_{u}}{1+\kappa+\gamma^{2} \tau_{v} \tau_{u}} E_{2}\left[\theta_{2}\right]+\frac{1+\kappa}{\gamma \tau_{v}} \theta_{2} .
$$

Given that $a_{2}=0, z_{2}=u_{2}$, and since traders at time 2 have also observed $z_{1}=\theta_{1}$, the second period stock of noise $\theta_{2}=\beta \theta_{1}+u_{2}$ can be exactly determined, and

$$
E_{2}\left[\theta_{2}\right]=\theta_{2}
$$

Hence, as argued above, traders perfectly anticipate the noise shock and accommodate it, and the price only reflects noise. But then this implies that

$$
p_{2}=\bar{v}+\frac{(\beta-1)(1+\kappa) \gamma \tau_{u}}{1+\kappa+\gamma^{2} \tau_{v} \tau_{u}} \theta_{2}+\frac{1+\kappa}{\gamma \tau_{v}} \theta_{2} .
$$

As a last step we need to characterize the first period equilibrium. Substituting the second period optimal strategy in the corresponding objective function and rearranging, at time 1 a trader chooses $x_{i 1}$ to maximize

$$
-E_{i 1}\left[\exp \left\{-\frac{1}{\gamma}\left(\left(p_{2}-p_{1}\right) x_{i 1}+\frac{(1+\kappa)\left(1+\kappa+\gamma \beta^{2} \tau_{v} \tau_{u}\right)}{2 \gamma \tau_{v}\left(1+\kappa+\gamma^{2} \tau_{v} \tau_{u}\right)} \theta_{2}^{2}\right)\right\}\right] .
$$

According to (85) $p_{2}$ only depends on $\theta_{2}$. Hence, in the first period the argument of the trader's objective function is a quadratic form of the random variable $\theta_{2}$ which is normally distributed:

$$
\theta_{2}\left|\theta_{1} \sim N\left(\beta \theta_{1}, \tau_{u}^{-1}\right) \Rightarrow\left(\theta_{2}-\beta \theta_{1}\right)\right| \theta_{1} \sim N\left(0, \tau_{u}^{-1}\right),
$$

and we can apply the usual transformation to compute the above expectation, obtaining that the function maximized by the trader is given by

$$
\left(\bar{v}-p_{1}\right) x_{i 1}+\beta \theta_{1}\left(m_{1} x_{i 1}+m_{2} \beta \theta_{1}\right)-\frac{1}{2 \gamma\left(\tau_{u}+(2 / \gamma) m_{2}\right)}\left(m_{1} x_{i 1}+2 m_{2} \beta \theta_{1}\right)^{2},
$$

where

$$
m_{1}=\frac{\left(1+\kappa+\gamma^{2} \beta \tau_{u} \tau_{v}\right)(1+\kappa)}{\left(1+\kappa+\gamma^{2} \tau_{u} \tau_{v}\right) \gamma \tau_{v}}, m_{2}=\frac{\left(1+\kappa+\gamma^{2} \beta^{2} \tau_{u} \tau_{v}\right)(1+\kappa)}{\left(1+\kappa+\gamma^{2} \tau_{u} \tau_{v}\right) 2 \gamma \tau_{v}} .
$$

Computing the first order condition and solving for $x_{i 1}$ yields

$$
\begin{aligned}
& X_{1}\left(p_{1}\right)=\frac{\gamma \tau_{v}}{1+\kappa}\left(\bar{v}-p_{1}\right) \\
& \quad+\frac{(\beta-1)\left(\left(1+\kappa+\gamma^{2} \tau_{u} \tau_{v}\right)\left(1+\kappa+\gamma^{2} \tau_{u} \tau_{v}(1+\beta)\right)+(1+\kappa)^{2}(1-\beta)\right)}{(1+\kappa)\left(1+\kappa+\beta \gamma^{2} \tau_{u} \tau_{v}\right)\left(\left(1+\kappa+\gamma^{2} \beta \tau_{u} \tau_{v}\right)^{2}+\beta \gamma^{4} \tau_{u}^{2} \tau_{v}^{2}(1-\beta)\right)}\left(p_{1}-\bar{v}\right) .
\end{aligned}
$$

Imposing market clearing and explicitly solving for the price

$$
p_{1}=\bar{v}+\Lambda_{1} \theta_{1}
$$

where

$$
\Lambda_{1}=\left(\frac{\gamma \tau_{v}}{1+\kappa}-\frac{(\beta-1)\left(\left(1+\kappa+\gamma^{2} \tau_{u} \tau_{v}\right)\left(1+\kappa+\gamma^{2} \tau_{u} \tau_{v}(1+\beta)\right)+(1+\kappa)^{2}(1-\beta)\right)}{(1+\kappa)\left(1+\kappa+\beta \gamma^{2} \tau_{u} \tau_{v}\right)\left(\left(1+\kappa+\gamma^{2} \beta \tau_{u} \tau_{v}\right)^{2}+\beta \gamma^{4} \tau_{u}^{2} \tau_{v}^{2}(1-\beta)\right)}\right)^{-1},
$$

which can be rearranged to obtain (27). 


\section{Proof of Proposition 3}

Follows immediately from the definition of equations (60), and (78).

QED

\section{Proof of Corollary 5}

Note that for $\kappa=0$ (41) and (56) imply $a_{n}=\gamma\left(\sum_{t=1}^{n} \tau_{\epsilon_{t}}\right)$, for $n=2,3$. Hence, $\rho_{2}=1$ and (66), (60) respectively become:

$$
\begin{aligned}
\lambda_{2} & =\frac{1+\gamma \tau_{u} \Delta a_{2}}{\gamma \tau_{2}+a_{2}}+\frac{(\beta-1) \tau_{u}\left(\gamma \tau_{2}+\beta a_{2}\right)\left(\tau_{2}-a_{2} \Delta a_{2} \tau_{u}\right)}{\tau_{i 2} D_{2}} \\
\alpha_{P_{2}} & =\alpha_{E_{2}}\left(1+\frac{(\beta-1) \gamma \tau_{2} \tau_{u}\left(\gamma \tau_{2}+\beta a_{2}\right)}{D_{2}}\right),
\end{aligned}
$$

so that

$$
\Upsilon_{2}^{1}=\frac{\gamma \tau_{2} \tau_{u}\left(\gamma \tau_{2}+\beta a_{2}\right)}{D_{2}}>0
$$

In the first period tedious algebra allows to show that

$$
h_{1,12}=-\frac{\lambda_{2} \tau_{i 2}^{2}\left(1-\lambda_{2} \gamma \tau_{\epsilon_{2}}\right)}{D_{1}}, \quad h_{1,22}=\frac{\left(\lambda_{2} \tau_{i 2}\right)^{2}}{D_{1}}
$$

where

$$
D_{1}=\tau_{i 2}^{2}\left(\lambda_{2}^{2} \tau_{i 1}+\left(1-\lambda_{2} \Delta a_{2}\right)^{2} \tau_{u}+\frac{(\beta-1)^{2}\left(\tau_{2}-a_{2} \Delta a_{2} \tau_{u}\right)^{2} \tau_{u} h_{2,22}}{D_{2}}\right) .
$$

Substituting (71), (72), and (91) in (74) and rearranging yields:

$$
\begin{aligned}
X_{1}\left(s_{i 1}, p_{1}\right)=\frac{a_{1}}{\alpha_{E_{1}}}\left(E_{i 1}[v]-\right. & \left.p_{1}\right)+\frac{\gamma}{h_{1,22}}\left(1-\tau_{i 1} h_{1,22}\right)\left(\hat{p}_{1}-p_{1}\right) \\
& -\frac{\gamma \tau_{u} \tau_{1} \beta(\beta-1)^{2}\left(\tau_{2}-a_{2} \Delta a_{2} \tau_{u}\right)\left(\gamma \tau_{i 3} \lambda_{3}\right)^{2}}{\lambda_{2}\left(1-\lambda_{2} \Delta a_{2}\right) D_{2}^{2}} E_{1}\left[\theta_{1}\right] .
\end{aligned}
$$

Using (91), we can now simplify (81) to obtain

$$
\begin{aligned}
a_{1}= & \frac{\tau_{\epsilon_{1}}}{\lambda_{2} \tau_{i 1} \tau_{i 2}}\left(\frac{D_{1} \Delta a_{2}}{\tau_{i 2}}-\left(\gamma \tau_{1}+\beta a_{1}\right)\left(\Delta a_{2} \tau_{u}\left(1-\lambda_{2} \Delta a_{2}\right)-\lambda_{2} \tau_{i 1}\right)+\right. \\
& \left.-\frac{(1-\beta)\left(\tau_{2}-a_{2} \Delta a_{2} \tau_{u}\right) \Delta a_{2} \tau_{u}\left(\gamma(1-\beta)\left(\tau_{2}-a_{2} \Delta a_{2} \tau_{u}\right)-\left(\gamma \tau_{2}+\beta a_{2}\right)\left(1-\lambda_{2} \gamma \tau_{\epsilon_{2}}\right)\right)}{D_{2}}\right) \\
= & \gamma \tau_{\epsilon_{1}},
\end{aligned}
$$

since, as one can verify,

$$
\begin{aligned}
\frac{D_{1}}{\tau_{i 2}}= & \lambda_{2} \tau_{i 1}\left(1+\gamma \Delta a_{2} \tau_{u}\right)+\left(1-\lambda_{2} \Delta a_{2}\right) \tau_{u}\left(\gamma \tau_{1}+\beta a_{1}\right)+ \\
& +\frac{(1-\beta)\left(\tau_{2}-a_{2} \Delta a_{2} \tau_{u}\right) \Delta a_{2} \tau_{u}\left(\gamma(1-\beta)\left(\tau_{2}-a_{2} \Delta a_{2} \tau_{u}\right)-\left(\gamma \tau_{2}+\beta a_{2}\right)\left(1-\lambda_{2} \gamma \tau_{\epsilon_{2}}\right)\right)}{D_{2}} .
\end{aligned}
$$

Finally, imposing market clearing yields

$$
\begin{aligned}
& \frac{(\beta-1) \tau_{1}}{1-\lambda_{2} \Delta a_{2}}\left(\frac{\alpha_{P_{2}}(\beta-1)\left(1-\alpha_{E_{2}}\right)+\alpha_{P_{2}}-\alpha_{E_{2}}}{\alpha_{E_{2}} \tau_{2}(\beta-1)}\right. \\
& \left.+\frac{\beta(\beta-1) \gamma \tau_{u}\left(\tau_{2}-a_{2} \Delta a_{2} \tau_{u}\right)\left(\gamma \tau_{i 3} \lambda_{3}\right)^{2}}{\lambda_{2} D_{2}^{2}}\right) E_{1}\left[\theta_{1}\right]=\frac{\gamma}{h_{1,22}}\left(\hat{p}_{1}-p_{1}\right) .
\end{aligned}
$$


We can now substitute (95) in (93). Imposing market clearing and rearranging allows to obtain an expression for the first period price as (77), where

$$
\begin{aligned}
\alpha_{P_{1}} & =\alpha_{E_{1}}\left\{1+\frac{(\beta-1) \gamma \tau_{1}\left(1-h_{1,22} \tau_{i 1}\right)}{1-\lambda_{2} \Delta a_{2}} \frac{\alpha_{P_{2}}}{a_{2}}+\frac{(\beta-1) \tau_{1}}{1-\lambda_{2} \Delta a_{2}} \times\right. \\
& \left.\left(h_{1,22} \tau_{i 1} \frac{\beta(1-\beta) \gamma \tau_{u}\left(\tau_{2}-a_{2} \Delta a_{2} \tau_{u}\right)\left(\gamma \tau_{i 3} \lambda_{3}\right)^{2}}{\lambda_{2} D_{2}^{2}}+\left(1-h_{1,22} \tau_{i 1}\right) \frac{\alpha_{P_{2}}-\alpha_{E_{2}}}{\alpha_{E_{2}} \tau_{2}(\beta-1)}\right)\right\} .
\end{aligned}
$$

Finally, for $\alpha_{P_{2}}$, using (90), the result stated in the corollary is immediate. For $\alpha_{P_{1}}$, inspection of (96) shows that $\alpha_{P_{1}}<\alpha_{E_{1}}$ if and only if $\beta<1$ since the sum of the terms multiplying $\beta-1$ :

$$
\begin{aligned}
& \Upsilon_{1}^{1}+\Upsilon_{1}^{2}=\frac{\gamma \tau_{1}\left(1-h_{1,22} \tau_{i 1}\right)}{1-\lambda_{2} \Delta a_{2}} \frac{\alpha_{P_{2}}}{a_{2}}+\frac{\tau_{1}}{1-\lambda_{2} \Delta a_{2}} \times \\
& \quad\left(h_{1,22} \tau_{i 1} \frac{\beta(1-\beta) \gamma \tau_{u}\left(\tau_{2}-a_{2} \Delta a_{2} \tau_{u}\right)\left(\gamma \tau_{i 3} \lambda_{3}\right)^{2}}{\lambda_{2} D_{2}^{2}}+\left(1-h_{1,22} \tau_{i 1}\right) \frac{\alpha_{P_{2}}-\alpha_{E_{2}}}{\alpha_{E_{2}} \tau_{2}(\beta-1)}\right),
\end{aligned}
$$

can be verified to be always positive.

QED

\section{Proof of COROLlary 8}

For the first part of the corollary, consider the following argument. From the first order condition of the trader's problem in the second period

$$
x_{i 2}=\gamma \frac{E_{i 2}\left[p_{3}-p_{2}\right]}{h_{2,22}}-\frac{h_{2,21}(1+\kappa)}{\gamma h_{2,22}} E_{i 2}\left[x_{i 3}\right] .
$$

Imposing market clearing, using (48) and (49), and rearranging yields

$$
\begin{aligned}
\frac{\tau_{2}\left(\beta \rho_{2}-1\right)}{h_{2,22} \tau_{i 3}\left(1-\lambda_{3} \Delta a_{3}\right)}\left(h_{2,22}-\frac{\lambda_{3} \Delta a_{3}(1+\kappa)}{\rho_{2} \tau_{i 2}}\right) E_{2}\left[\theta_{2}\right]-\frac{h_{2,21}(1+\kappa)\left(1-\alpha_{E_{2}}\right)\left(1-\beta \rho_{2}\right)}{\gamma h_{2,22} \rho_{2} \tau_{i 2} \tau_{i 3}} E_{2}\left[\theta_{2}\right] \\
+\left(1+\frac{\alpha_{E_{2}}}{a_{2}}\left(\frac{h_{2,21}(1+\kappa) a_{3}\left(1-\lambda_{3} \Delta a_{3}\right)}{\gamma h_{2,22} \tau_{i 3} \alpha_{E_{3}}}-\frac{\gamma \lambda_{3} \Delta a_{3}}{h_{2,22}}\right)\right) \theta_{2}=0 .
\end{aligned}
$$

The first line in the above equation respectively captures the impact that the expected change in price and the expected third period position have on traders' aggregate second period strategy. Rearranging the term multiplying $\theta_{2}$ in the second line yields

$$
\begin{aligned}
1+\frac{\alpha_{E_{2}}}{a_{2}}\left(\frac{h_{2,21}(1+\kappa) a_{3}\left(1-\lambda_{3} \Delta a_{3}\right)}{\gamma h_{2,22} \tau_{i 3} \alpha_{E_{3}}}-\frac{\gamma \lambda_{3} \Delta a_{3}}{h_{2,22}}\right) & =1+\frac{\alpha_{E_{2}}}{a_{2}}\left(-\frac{a_{2}}{\alpha_{E_{2}}}\right) \\
& =0 .
\end{aligned}
$$

The above result implies that for any realization of $E\left[E_{2}\left[\theta_{2}\right] \mid v\right]=\left(a_{2} / \alpha_{P_{2}}\right) E\left[p_{2}-E_{2}[v] \mid v\right]$,

$$
\frac{\tau_{2}\left(\beta \rho_{2}-1\right)}{h_{2,22} \tau_{i 3}\left(1-\lambda_{3} \Delta a_{3}\right)}\left(h_{2,22}-\frac{\lambda_{3} \Delta a_{3}(1+\kappa)}{\rho_{2} \tau_{i 2}}\right) E\left[E_{2}\left[\theta_{2}\right] \mid v\right]
$$

and

$$
-\frac{h_{2,21}(1+\kappa)\left(1-\alpha_{E_{2}}\right)\left(1-\beta \rho_{2}\right)}{\gamma h_{2,22} \rho_{2} \tau_{i 2} \tau_{i 3}} E\left[E_{2}\left[\theta_{2}\right] \mid v\right]
$$


must have opposite sign. Given that $h_{2,21}$ can be verified to be negative, this implies that if (and only if) $\beta \rho_{2}>1, E\left[\bar{E}_{2}\left[p_{3}-p_{2}\right] \mid v\right]$ is positive. If $\kappa=0$, then a similar argument shows that at time $2 E\left[p_{2}-E_{2}[v] \mid v\right]<0 \Leftrightarrow E\left[\bar{E}_{2}\left[p_{3}-p_{2}\right] \mid v\right]>0$ for $\beta<1$.

In the absence of residual uncertainty, at time $n=1$, using (95) and rearranging the market clearing equation yields

$$
\begin{array}{r}
\frac{h_{1,22}}{\gamma} \frac{(\beta-1) \tau_{1}}{1-\lambda_{2} \Delta a_{2}}\left(\frac{\alpha_{P_{2}}(\beta-1)\left(1-\alpha_{E_{2}}\right)+\alpha_{P_{2}}-\alpha_{E_{2}}}{\alpha_{E_{2}} \tau_{2}(\beta-1)}\right. \\
\left.+\frac{\beta(\beta-1) \gamma \tau_{u}\left(\tau_{2}-a_{2} \Delta a_{2} \tau_{u}\right)\left(\gamma \tau_{i 3} \lambda_{3}\right)^{2}}{\lambda_{2} D_{2}^{2}}\right) E_{1}\left[\theta_{1}\right]=\hat{p}_{1}-p_{1} .
\end{array}
$$

Averaging out noise in the above expression, in this case the sign of $E\left[\bar{E}_{1}\left[p_{2}-p_{1}\right] \mid v\right]$ depends on the sign of the sum of the term multiplying $E\left[E_{1}\left[\theta_{1}\right] \mid v\right]$ in the above expression and

$$
\lambda_{2} \Delta a_{2}\left(\frac{\alpha_{E_{1}}}{a_{1}}-\frac{\beta \alpha_{P_{2}}}{a_{2}\left(1-\lambda_{2} \Delta a_{2}\right)}\right),
$$

which after rearranging can be shown to be always negative provided $\beta<1$. 